\title{
Substrate Stiffness-Dependent Regulatory Volume Decrease and Calcium Signaling in Chondrocytes
}

Quanyou Zhang ( $\sim$ zhangquanyou@tyut.edu.cn )

Shanxi Medical University

Min Zhang

Taiyuan University of Technology

Xiaoan Wu

University of Miami

Genlai Du

Shanxi Medical University

Xiaochun Wei

Shanxi Medical University

Weiyi Chen

Taiyuan University of Technology

\section{Research Article}

Keywords: substrate stiffness, regulatory volume decrease, viscoelasticity, calcium signaling

Posted Date: December 10th, 2020

DOl: https://doi.org/10.21203/rs.3.rs-114377/v1

License: (c) (1) This work is licensed under a Creative Commons Attribution 4.0 International License. Read Full License

Version of Record: A version of this preprint was published at Acta Biochimica et Biophysica Sinica on November 1st, 2021. See the published version at https://doi.org/10.3724/abbs.2021008. 


\section{Abstract}

The pericellular matrix stiffness is strongly associated with its biochemical and structural changes During the aging and osteoarthritis progresses of articular cartilage. However, how substrate stiffness modulates the chondrocyte regulatory volume decrease (RVD) and calcium signaling remains unknown. This study aims to investigate the effects of substrate stiffness on the chondrocyte RVD and calcium signaling by recapitulating the physiologically relevant substrate stiffness. Our results showed that substrate stiffness induced completely different dynamical deformation between the cell swelling and recovering progresses. Chondrocytes swelled faster on the soft substrate but recovered slower than the stiff substrate during the RVD response induced by the hypo-osmotic challenge. We found that stiff substrate enhanced the cytosolic $\mathrm{Ca}^{2+}$ oscillation of chondrocytes in the iso-osmotic medium. More importantly, chondrocytes exhibited a distinctive cytosolic $\mathrm{Ca}^{2+}$ oscillation during the RVD response. Soft substrate significantly improved the $\mathrm{Ca}^{2+}$ oscillation during the cell swelling whereas stiff substrate enhanced the cytosolic $\mathrm{Ca}^{2+}$ oscillation during the cell recovering. Our work also suggests that TRPV4 channel are involved in the chondrocyte sensing substrate stiffness and RVD response by mediating $\mathrm{Ca}^{2+}$ signaling in a stiffness-dependent manner. It helps to understand a previously unidentified relationship between substrate stiffness and RVD response under the hypo-osmotic challenge.

\section{Introduction}

Chondrocytes are the solitary cell type of articular cartilage and responsible for the slow turnover of matrix [1]. The pericellular matrix (PCM) with defined physical properties surrounds each chondrocyte [2] and plays a critical role in regulating the mechanical microenvironment of chondrocytes $[3,4]$. During the aging and osteoarthritis (OA) progresses of articular cartilage, the mechanical properties (i.e. stiffness) of the PCM can markedly changes between $1 \mathrm{kPa}$ and $205 \mathrm{kPa}$ [5-8]. One study has shown that the PCM stiffness is reduced by $30-50 \%$ in the development of OA [9]. The reduction of PCM stiffness induced by OA modulates chondrocyte function-related spatial organization and biosynthetic activity $[10,11]$. Furthermore, the loss of PCM stiffness corresponds to the increased extent of cell swelling and calcium signaling mediated by transient receptor potential vanilloid channel 4 (TRPV4) in response to osmotic stress $[12,13]$. Cell volume regulation is important to chondrocyte physiology. Under the hypo-osmotic stimulus, chondrocyte volume rapidly increases, then followed by active regulatory volume decrease (RVD) which exhibit an active volume recovery process [14]. However, how changes in PCM stiffness influence the RVD and TRPV4-mediated calcium signaling in chondrocytes remains unknown.

As a key physical factor of cell microenvironment, cell-supporting substrate stiffness is well recognized to regulate diverse cell functions [15]. Substrate stiffness has been shown to modulate the chondrocyte morphology, phenotype and mechanical behavior $[16,17]$. The RVD is crucial for many biophysical and biological responses of chondrocytes. A loss of chondrocyte RVD is associated with cell death and progression of OA $[18,19]$. Changes to volume affect the phenotypic plasticity and matrix metabolism of chondrocytes $[20,21]$. For example, RVD-induced changes in cell shape can affect membrane transporter 
activity potentially mediated via $\mathrm{Ca}^{2+}$ signaling. TRPV4, the primary regulator of $\mathrm{Ca}^{2+}$ signaling, has been proposed as a mechanosensitive channel that is involved in regulating the mechanotransduction and RVD response of chondrocytes [22-27]. It is assumed to involve in cell sensing substrate stiffness by locally altering the $\mathrm{Ca}^{2+}$ permeability $[28,29]$. Since calcium signaling plays an important role in multiple signaling pathways and mechanotransduction in chondrocytes [30,31], substrate stiffness may modulate the chondrocyte mechanotransduction via the TRPV4-mediated $\mathrm{Ca}^{2+}$ signaling, which eventually affects cell function [15]. Collectively, these findings highlight the potential role of substrate stiffness in regulating the RVD response and TRPV4-mediated calcium signaling in chondrocytes. Previous studies have suggested that PCM stiffness affects the chondrocyte volume swelling and TRPV4-mediated $\mathrm{Ca}^{2+}$ signaling [13]. However, these studies normally have been focused on the swelling process but neglected the dynamic recovering process of chondrocytes. To date, neither theoretical nor experimental studies have been focused on the role of substrate stiffness in the RVD response and TRPV4-mediated $\mathrm{Ca}^{2+}$ oscillations during the chondrocyte swelling and recovering processes.

Taken together, in this study, we hypothesize (I) that substrate stiffness modulates the mechanical properties of chondrocytes, resulting in the substrate stiffness-dependent RVD response, and (II) that TRPV4 channels are involved in chondrocytes sensing substrate stiffness by mediating cytosolic $\mathrm{Ca}^{2+}$ signaling. To test these hypotheses, Polydimethylsiloxane (PDMS) was utilized to engineer cellsupporting substrate with physiologically-relevant stiffness. The mechanical properties of chondrocytes were evaluated by using atomic force microscopy (AFM). $\mathrm{Ca}^{2+}$ oscillation of chondrocytes cultured on variable stiffness substrates was measured by using fluorescence confocal laser scanning microscopy and Fluo-4 AM ratiometric method.

\section{Materials And Methods}

\subsection{Preparation of substrates with different stiffness}

PDMS substrate stiffness was formulated to mimic the physiologically-related PCM elasticity that was either stiff $(2.2 \pm 0.3 \mathrm{MPa})$, medium $(46.5 \pm 5.8 \mathrm{kPa})$ and soft $(2.1 \pm 0.2 \mathrm{kPa})$, respectively. Briefly, PDMS curing agent (Sylgard184, Dow corning Corp) was mixed with a base agent in a mass ratio of 1:10 (stiff), 1:50 (medium) and 1:70 (soft), respectively. The mixture was poured into $35-\mathrm{mm}$ petri dishes to create 1$\mathrm{mm}$ thick films and then cured at $70{ }^{\circ} \mathrm{C}$ for 6 hours. After curing, these PDMS substrates were placed in an oxygen plasma cleaner (SBC-12, KYKY) for surface oxidation. The stiffness of PDMS substrate (elastic modulus) was determined using the indentation method and ElectroForceH3100 test instrument (Bose, Shanghai, China) as previously described [32]. Before seeding cells, substrates with varying stiffness were coated by $0.02 \mathrm{mg} / \mathrm{ml}$ rat type I collagen (Shengyou Biotechnology) at $4^{\circ} \mathrm{C}$ overnight and sterilized by UV radiation for 45 minutes, as previously described [17].

\subsection{Isolation of primary chondrocytes}


A total of 24 animals of $5 \sim 6$ day old mice (C57BL/6) were used in this study. All animal procedures were approved by the Animal Ethics committee of Taiyuan University of Technology and conducted in accordance with international standards on animal welfare. Mice were killed under general anesthesia. Femoral condyles and tibial plateau were isolated from the hind legs using scissors and pincer. The pieces of cartilage were incubated in the collagenase $D$ digestion solution $(3 \mathrm{mg} / \mathrm{ml})$ for $45 \mathrm{~min}$ at $37^{\circ} \mathrm{C}$ in a thermal incubator under $5 \% \mathrm{CO}_{2}$. Then the cartilage pieces were placed in a new Petri dish with collagenase $\mathrm{D}$ solution $(0.5 \mathrm{mg} / \mathrm{ml})$ overnight at $37^{\circ} \mathrm{C}$, as previously described [33]. Cells were isolated by overnight incubation and filtered through a $40 \mathrm{~mm}$ cell strainer (BD-Falcon). The resulting cell suspension was washed twice in fresh Dulbecco's modified eagle's medium (DMEM) $11 \mathrm{~g}$ glucose per ml; no Lglutamine $\mathrm{pH}$ 7.0-7.6, Osmolality 304-336 mos/kg, Sigma, USA). Cells were re-suspended in DMEM supplemented with $10 \%$ fetal calf serum (Sigma) and $1 \%$ penicillin/streptomycin solution at the density of $8^{\prime} 10^{3}$ cells $/ \mathrm{cm}^{2}$ (the final osmolarity is $320 \mathrm{mOsm}$ ). Cells were grown on substrates of varying stiffness at $37^{\circ} \mathrm{C}$ in $5 \% \mathrm{CO}_{2}$. In AFM experiments, chondrocytes were rinsed with phosphate buffered saline (PBS) solution to avoid proteins and cellular debris adhesive to tip prior to single-cell stress relaxation tests. In the RVD experiments, chondrocytes cultured on varying substrate stiffness were exposed to hypo-osmotic medium $(180 \mathrm{mOsm})$. All experiments were performed at $37^{\circ} \mathrm{C}$ and controlled humidity (custom-built equipment) within $1.5 \mathrm{~h}$ unless otherwise stated.

\subsection{Cell diameter change during hypo-osmotic (180 mOsm) induced RVD response}

The chondrocytes were cultured for 24 hours in the iso-osmotic medium (320 mOsm) before exposure to the 180 mOsm medium. The complete cell RVD response suggests that following hypo-osmotic challenge, cells initially swell passively, and then volume recovery. Cells were considered to show RVD response following hypo-osmotic $(180 \mathrm{mOsm})$ if the cell diameter reduced by at least $1 \mu \mathrm{m}$ during the imaging period [34]. The whole process of RVD response was tracked. Bright-field sequential time-lapse images of chondrocytes on substrates of varying stiffness were obtained every $10 \mathrm{~s}$ for $45 \mathrm{~min}$ by microscopy (FV1000, Olympus) with a 40' oil immersion objective lens. The diameters $d$ of single cells were quantitatively calculated from the mean of the two orthogonal diameters measured using ImageJ software. Then, we analyzed the following parameters. The swelling time, $T_{S}$, is the time the cell takes to swell until it reaches its maximum cell diameter. In contrast, the recovering time, $T_{R}$, is the time the cell takes to recover from its maximum cell diameter. The responding time, $T_{\text {Res, }}$ is the entire time during the whole RVD process which equals the sum of $T_{S}$ and $T_{R}$. The cell diameter rate, $V_{d}$, is the change of diameter over time during the RVD response.

\subsection{AFM testing}


In AFM experiments, we only focused on the changes in the mechanical properties of chondrocytes on substrates of varying stiffness during the cell swelling. The mechanical properties of single chondrocyte were measured by an AFM (NTEGRA Solaris, NT-MDT, Russia) combined with a phase contrast microscopy (IX-70, Olympus). Chondrocytes were tested at a density of $2^{\prime} 10^{4} \mathrm{cells} / \mathrm{cm}^{2}$, so that individual cells would not be connected with others. A gold-coated, 5-mm diameter, spherical tip cantilevers (spring constant is $0.05 \mathrm{~N} / \mathrm{m}$, Novascan Technologies, Inc.) was used for indentation and stress-relaxation experiments. Indentations were applied with a force trigger of $2.5 \mathrm{nN}$. An approach velocity of $6.53 \mu \mathrm{m} / \mathrm{s}$ was used and followed by a 100 -s relaxation period. A constant displacement of about $2 \mu \mathrm{m}$ indentation was hold for all the stress-relaxation experiments. The elastic modulus and viscoelastic parameters of chondrocyte were determined according to a modified Hertz equation (Eq.1) and a stress relaxation model of viscoelastic solid (Eq.2), respectively. They are given by [35],

$$
\left.\begin{array}{c}
F(\delta)=\frac{4 R^{\frac{1}{2}} E_{\text {elstis }}}{3\left(1-v^{2}\right)} \delta^{\frac{3}{2}} C \quad R=\left(\frac{1}{R_{\text {prose }}}+\frac{1}{h / 2}\right)^{-1} \\
F(t)=\frac{4 R^{\frac{1}{2}} \delta_{0}^{\frac{3}{2}} E_{R}}{3(1-v)}\left(1+\frac{\tau_{\sigma}-\tau_{z}}{\tau_{z}} e^{-t / z_{z}}\right) C \\
E_{0}=E_{R}\left(1+\frac{\tau_{\sigma}-\tau_{z}}{\tau_{z}}\right), \mu=E_{R}\left(\tau_{\sigma}-\tau_{z}\right)
\end{array}\right\}
$$

Where $F$ is the applied force; $R$ is the relative radius, $\mathrm{n}$ is the Poisson's ratio which is assumed to be $0.5, \mathrm{C}$ is a constant depending only on the indentation during stress relaxation. The elastic modulus, $E_{\text {elastic, }}$ is determined by fitting the indentation approach curves with Hertz equation (Eq.1). $d$ is the indentation depth. Data from the relaxation phase of the test were fit with the viscoelastic equation (Eq. 2) to determine the $E_{\mathrm{R}}, \tau_{\sigma}$, and $\tau_{\varepsilon}$. $E_{0}$ and $\mathrm{m}$ were calculated using Eq. 2. $E_{\mathrm{R}}$ and $E_{0}$ are instantaneous modulus and relaxed modulus, respectively. $\tau_{\sigma}$ represents the time of relaxation of deformation under constant load, $\tau_{\varepsilon}$ represents the time of relaxation of load under constant deformation. $m$ is the apparent viscosity. All AFM experiments were carried out at $37^{\circ} \mathrm{C}$ and controlled humidity, as previously described [17].

\subsection{Calcium imaging}

Chondrocytes on substrates of varying stiffness were loaded with Fluo-4 AM (Life Technology) in pure DMEM buffer for $35 \mathrm{~min}$ at $37^{\circ} \mathrm{C}$ before imaging. To modulate the TRPV4 channel activity, we applied 10 $\mu \mathrm{M}$ GSK205 or $1 \mu \mathrm{M}$ 4aPDD (Merck Millipore) to the bath solution. GSK205 is a TRPV4 selective antagonist while 4aPDD is a TRPV4 selective agonist. $\mathrm{Ca}^{2+}$ signaling imaging of chondrocytes was recorded in response to substrates of varying stiffness and hypo-osmotic $(180 \mathrm{mOsm})$ challenge by using fluorescence confocal laser scanning microscopy (FV1000, Olympus). Time-series images of baseline $\mathrm{Ca}^{2+}$ oscillations were recorded every $3 \mathrm{~s}$ for a total of $15 \mathrm{~min}$ and fluorescence was analyzed by 
using the ImageJ software. The difference $(\Delta F)$ between the mean fluorescence measured in a given region of interest $(\mathrm{ROI})$ and the corresponding control value for each $\mathrm{ROI}\left(F_{0}\right)$ was expressed as the fraction of the control $\left(\Delta F / F_{0}\right)$ and then plotted as a function of time. In the RVD experiments, we simultaneously monitored the cell diameter change and chondrocyte $\mathrm{Ca}^{2+}$ oscillations. A cell was defined as responsive if it showed a calcium peak with a magnitude four times higher than its maximum fluctuation along the baseline [36].

\subsection{Statistical Analysis}

Statistical significance between the groups was estimated using one-way analysis of variance (ANOVA) followed by a Tukey post hoc test using Origin (OriginLab, Northampton, MA). P values less than 0.05 are considered to be significant. All data are presented as mean \pm standard deviation.

\section{Results}

\subsection{Substrate stiffness affects the RVD response of chondrocytes}

Our results showed that the RVD response of chondrocytes was intimately related to substrate stiffness. The soft substrate markedly increased the percentage of RVD response in chondrocytes $(p<0.001)$ (Fig.1A). Substrate stiffness caused distinctive swelling and recovering behaviors of chondrocytes (Fig. 1B). The change of cell diameter $d$ depended on the substrate stiffness during the RVD response (Fig.1C). The trend of non-dimensional diameter $d / d_{0}$ ( $d_{0}$ is the initial cell diameter) over time remained the same with Fig.1C, and it was independent of the cell's initial diameter (Fig.1D). We found that stiff substrate significantly prolonged the swelling time $T_{S}$ of chondrocytes $(p<0.001)$ (Supplementary Table I and Fig.1E), and soft substrate significantly increased the percentage increase of diameter in chondrocytes during the cell swelling $(p<0.001)$ (Fig.1F). In contrast, during the cell recovery, the recovery time $T_{R}$ in chondrocytes on the stiff substrate was significantly shorter than that on the soft substrate $(p<0.05)$ (Fig.1G). Furthermore, stiff substrate can obviously enhance the capacity of cell diameter recovery during cell recovering $(p<0.01)($ Fig. $1 \mathrm{H})$. Interestingly, the trend of the swelling and recovering time was completely opposite to that of the percentage increase of diameter in chondrocytes during cell RVD responding. Lastly, the soft substrate significantly increased the mean RVD responding time $T_{\text {Res }}$ of chondrocytes $(p<0.01)$ (Fig.1I).

The cell diameter rate was obtained by fitting the cell diameter vs. time curves from substrates of varying stiffness. The cell diameter vs. time curves from all substrates during the cell swelling exhibited an approximately linear relationships (Fig.2A-C). Moreover, the soft substrate markedly increased the cell diameter rate during the cell swelling caused by hypo-osmotic $(180 \mathrm{mOsm})$ medium $(p<0.001)$ (Fig.2D). Then, we fitted the approximately linear part of cell diameter vs. time curves during the cell recovering 
(Fig. 2E-G). However, stiff substrate significantly increased the recovering cell diameter rate $(p<0.001)$ (Fig.2H). For the first time, our results indicated that hypo-osmotic $(180 \mathrm{mOsm})$ challenge-induced chondrocyte deformation is substrate stiffness-dependent.

\subsection{Stiff substrate enhances the mechanical properties of chondrocytes during the cell swelling}

AFM experiments were used to measure the mechanical properties of chondrocytes on substrates of varying stiffness in the iso-osmotic (320 mOsm) and hypo-osmotic (180 mOsm) medium (Fig. 3A). When the constant indentation displacement $2 \mu \mathrm{m}$ was held on chondrocytes, chondrocytes first exhibited typical elastic response consistent with the Hertz model and then exhibited stress relaxation consistent with the viscoelastic theoretical model (Fig.3B). Theoretical model fitting of all experimental data exhibited excellent consistency for elastic and viscoelastic equations respectively (Fig. 3C). Our results indicated that stiff substrate enhances the elastic $\left(E_{\text {elastic }}\right)$ and viscoelastic parameters $\left(E_{\mathrm{R}}, E_{0}\right.$ and $\left.\mathrm{m}\right)$ of chondrocytes during the cell swelling in hypo-osmotic $(180 \mathrm{mOsm})$ medium $(p<0.001)$ (Table I and Fig. 3D-G).

Then we compared mechanical parameters of chondrocytes on variable stiffness substrates between the iso-osmotic (Supplementary Table II) and hypo-osmotic (180 mOsm) medium. Our results showed compared to iso-osmotic medium, chondrocytes on the stiff substrate during the cell swelling in the hypoosmotic $(180 \mathrm{mOsm})$ showed higher elastic and viscoelastic parameters including $E_{\text {elastic }}, E_{\mathrm{R}}, E_{0}$ and $\mathrm{m}$ $(\mathrm{p}<0.001)(\mathrm{Fig} .3 \mathrm{H}-\mathrm{K})$. Moreover, the mean percentage increases in elastic modulus $E_{\text {elastic }}$ of chondrocytes were higher on the soft substrate than that on the stiff substrate during the cell swelling. However, in response to the hypo-osmotic medium (180 mOsm), the mean percentage increases in viscoelastic parameters $\left(E_{\mathrm{R}}, E_{0}\right.$ and $\left.\mu\right)$ of chondrocytes were higher on the stiff substrate than those on the soft substrate (Supplementary Table III). Our results suggested that substrate stiffness determines regulates the dynamic elastic and viscoelastic mechanical properties of chondrocytes during the hypoosmotic (180 mOsm) challenge.

\subsection{Stiff substrate enhances cytosolic $\mathrm{Ca}^{2+}$ oscillation of chondrocytes in iso-osmotic medium}

Chondrocytes on variable stiffness substrates showed cytosolic $\mathrm{Ca}^{2+}$ oscillations in the iso-osmotic medium (320 mOsm) (Fig. 4A). Stiff substrate showed the greatest percentage of $\mathrm{Ca}^{2+}$ oscillations of chondrocytes $(p<0.001)$ (Fig. 4B). We recorded the amplitude and frequency of $\mathrm{Ca}^{2+}$ oscillation in chondrocyte on variable stiffness substrates (Fig. 4C, D). Our results showed that stiff substrate markedly enhanced both the amplitude and frequency of $\mathrm{Ca}^{2+}$ oscillations in chondrocytes ( $\left.p<0.001\right)$ (Fig. 4E, I). To test if TRPV4 channels are involved in substrate stiffness mediating $\mathrm{Ca}^{2+}$ signaling in chondrocytes, we 
applied TRPV4 channel activator 4aPDD and inhibitor GSK205 when recording $\mathrm{Ca}^{2+}$ oscillations, respectively. TRPV4 activator 4aPDD significantly enhanced the calcium responsive rate, amplitude and frequency of $\mathrm{Ca}^{2+}$ oscillations in chondrocytes on all substrates of varying stiffness while TRPV4 inhibitor GSK205 weakened those $\mathrm{Ca}^{2+}$ effects. ( $\left.p<0.01\right)$ (Fig. 4F-H, J-L, Supplementary Table IV). In addition, when treated with TRPV4 activator 4aPDD, chondrocytes on the stiff substrate showed the highest amplitude and frequency of $\mathrm{Ca}^{2+}$ oscillations $(p<0.001)$ (Fig. $\left.4 \mathrm{E}, \mathrm{I}\right)$. They also showed a bigger percentage increase of amplitude and frequency of $\mathrm{Ca}^{2+}$ oscillations than chondrocytes on the soft substrate (Supplementary Table V). On the other hand, when treated with the TRPV4 inhibitor GSK205, chondrocytes on varying substrates showed no significant difference in the amplitude of $\mathrm{Ca}^{2+}$ oscillations ( $p>0.25$ ) (Fig. $4 G$ ), though chondrocytes on the stiff substrate exhibited a higher frequency of $\mathrm{Ca}^{2+}$ oscillations $(p<0.05)$ (Fig. $\left.4 \mathrm{~J}\right)$. Chondrocytes showed a bigger percentage decrease of amplitude and frequency of $\mathrm{Ca}^{2+}$ oscillations on the stiff substrate than the soft substrate with the application of GSK205 (Supplementary Table V). Taken together, these results suggested that mechanosensitive TRPV4 channel is involved in chondrocytes sensing substrate stiffness and mediating the calcium signaling.

\subsection{Substrate stiffness affects the hypo-osmotic (180 mOsm) challenge-induced $\mathrm{Ca}^{2+}$ oscillations in chondrocytes}

Only chondrocytes that exhibited both RVD response and $\mathrm{Ca}^{2+}$ oscillations were used in this analysis. Our results show that soft and medium substrates significantly increased the percentage of chondrocytes exhibited both RVD response and $\mathrm{Ca}^{2+}$ oscillations ( $42 \pm 5 \%$ and $39 \pm 4 \%$, respectively) than the stiff substrate $(31 \pm 5 \%)(p<0.01)$. We then quantified the amplitude and frequency of $\mathrm{Ca}^{2+}$ oscillation in chondrocytes cultured on variable stiffness substrates during the cell swelling and recovering (Fig. 5A, B). The results showed that soft substrate induced a significantly higher percentage of $\mathrm{Ca}^{2+}$ oscillations (58 $\pm 7 \%$ ) in chondrocytes than medium $(77 \pm 8 \%)$ and stiff substrates $(79 \pm 8 \%)$ during the hypo-osmotic (180 mOsm) challenge $(p<0.001)$. Application of TRPV4 activator 4aPDD or the TRPV4 inhibitor GSK205 significantly enhanced or weakened the calcium responsive rate of $\mathrm{Ca}^{2+}$ oscillations in chondrocytes on variable stiffness substrates, respectively $(\mathrm{p}<0.01)$ (Supplementary Table $\mathrm{VI})$. During the cell swelling, the soft substrate enhanced both the amplitude and frequency of $\mathrm{Ca}^{2+}$ oscillations ( $\left.p<0.01\right)$ (Fig. 5C, F). With the treatment of TRPV4 activator 4aPDD, both the amplitude and frequency of $\mathrm{Ca}^{2+}$ oscillations in chondrocytes were significantly higher on soft substrate $(p<0.005)$ (Fig. 5D, G). With the treatment of TRPV4 inhibitor GSK205, the amplitude and frequency of $\mathrm{Ca}^{2+}$ oscillations were significantly higher on the stiff substrate $(p<0.05)$ (Fig. $5 \mathrm{E}, \mathrm{H})$. Moreover, the relative percentage increase of $\mathrm{Ca}^{2+}$ oscillations (amplitude and frequency) in chondrocytes treated with TRPV4 activator 4aPDD was higher on the soft substrate than the stiff substrate during the cell swelling. On the other hand, the decrease of $\mathrm{Ca}^{2+}$ 
oscillations induced by GSK205 was also higher on the soft substrate than the stiff substrate. (Supplementary Table VII).

During the cell recovering process, the stiff substrate enhanced both the amplitude and frequency of $\mathrm{Ca}^{2+}$ oscillations in chondrocytes $(p<0.001)$ (Fig. 5l, L). With the treatment of TRPV4 activator 4aPDD, both the amplitude and frequency of $\mathrm{Ca}^{2+}$ oscillations in chondrocytes were significantly higher on the stiff substrate $(p<0.01)$ (Fig. 5J, M). With the treatment of TRPV4 inhibitor GSK205, the amplitude of $\mathrm{Ca}^{2+}$ oscillations in chondrocytes showed no significant difference between varying substrates $(p>0.05)$ (Fig. $5 \mathrm{~K})$, but the frequency of $\mathrm{Ca}^{2+}$ oscillations in chondrocytes was significantly higher on the stiff substrate $(p<0.05)$ (Fig. 5N). Moreover, with the treatment of TRPV4 activator 4aPDD or the TRPV4 inhibitor GSK205, stiff substrate induced a higher percentage increase or decrease in $\mathrm{Ca}^{2+}$ oscillation in chondrocytes than the soft substrate (Supplementary Table VIII), respectively. Our results, therefore, suggested that the TRPV4 channel is involved in chondrocyte RVD response, exhibiting a completely different substrate stiffness-dependent mode of $\mathrm{Ca}^{2+}$ oscillations between the cell swelling and recovering progress.

\section{Discussion}

In this study, we only focused on the effects of the microenvironmental physical factor, substrate stiffness, on the RVD and calcium signaling in chondrocytes. Our results showed that substrate stiffness induced deformational discrepancies between the cell swelling and recovering under hypo-osmotic (180 mOsm) stimulus. Soft substrate induced robust swelling behaviors of chondrocytes while stiff substrate contributed to a faster recovery behavior of chondrocytes during RVD response (Fig. 2D, H). This is not surprising because chondrocytes on the stiff substrate have been shown to exhibit higher stiffness and lower cell height before the RVD response [16], which might make chondrocytes more difficult to swell in response to the 180 mOsm osmolarity. In contrast, the lower mechanical properties of chondrocytes on the soft substrate might make them easy to rapidly swell. But it is unclear yet why chondrocytes on the stiff substrates recover faster than ones on soft substrates. It is well known that stiff substrate induces cytoskeletal stiffening and hypo-osmotic $(180 \mathrm{mOsm})$ challenge induces chromatin condensation, both of which contribute to the cell stiffening $[34,37,38]$. Therefore, the higher increase in mechanical properties of chondrocytes on the stiff substrate may make them store higher cytoskeletal tension and more recovery energy. This would prevent chondrocytes from swelling but instead provide them with greater motility to recover. It has been suggested that cell stiffness decreases with increasing volume as cells are grown on a substrate with fixed stiffness [38], this is contrary to our finding that the mechanical parameters of chondrocytes on substrate of varying stiffness are all increased in varying degrees during hydro-osmotic (180 mOsm) challenge.

Previous studies have been focused on the inactive mechanical response of chondrocytes to external osmolarity or biochemical stimulus [34,39-41]. Under the two-dimensional (2D) substrate test conditions, the observed RVD response on substrates of varying stiffness is directly related to not only the substrate 
stiffness-induced adhesive force but also to applied hypo-osmotic (180 mOsm) challenge. Comparing non-dimensional mechanical parameters, $E_{\text {elastic, }} E_{\mathrm{R}}, E_{0}$ and $\mathrm{m}$ with cell diameter rate $\mathrm{V}_{\mathrm{d}}$, we found that the $V_{d}$ was negatively correlated with those substrate stiffness-dependent cell moduli during the cell swelling (Fig. 6A). We also found that both the amplitude and frequency of $\mathrm{Ca}^{2+}$ oscillations were negatively correlated with those parameters during the cell swelling (Fig. 6B).

Here, we show that stiff substrate enhanced $\mathrm{Ca}^{2+}$ oscillations of chondrocytes. Moreover, the $\mathrm{Ca}^{2+}$ oscillations of chondrocytes in the hypo-osmotic medium (180 mOsm) were more robust compared with those in the iso-osmotic medium. The phenomena may be due to the large amounts of membrane area are involved in membrane stretching during the hypo-osmotic $(180 \mathrm{mOsm})$ challenge. However, this explanation is only suitable for the cell swelling process but not for the cell recovering process. Unfortunately, no mechanism underlying this phenomenon is available.

We found that the amplitude and frequency of $\mathrm{Ca}^{2+}$ oscillations were consistently correlated with the change in $V_{d}$ during the cell swelling process (Fig. 6C, D). During cell recovering process, the amplitude of $\mathrm{Ca}^{2+}$ oscillations were not significantly consistently correlated with the change in $V_{\mathrm{d}}$ (Fig. 6E), but the frequency of $\mathrm{Ca}^{2+}$ oscillations were consistently correlated with the change in $V_{\mathrm{d}}$ (Fig. 6F). That suggests the relation between cell diameter rate $V_{\mathrm{d}}$ and $\mathrm{Ca}^{2+}$ oscillations during the RVD response contributes to elucidating the mechanism underlying the RVD response. The observed difference in $\mathrm{Ca}^{2+}$ oscillations of chondrocytes on variable stiffness substrates may have important implications for regulating cell function through the cell-instructive substrate or scaffold. For example, inappropriate increases in chondrocyte volume lead to the progression of OA [18]. The robust $\mathrm{Ca}^{2+}$ oscillations can enhance anabolic gene expression and matrix synthesis that are required to produce functional cartilage [27].

In vivo $\mathrm{PCM}$ may involve other mechanical and biochemical factors to co-regulate $\mathrm{Ca}^{2+}$ oscillations. Here, we only focus on the effects of 2D substrate stiffness on hypo-osmotic challenge-induced $\mathrm{Ca}^{2+}$ oscillations of chondrocytes. However, our recent study indicated that the more physically-relevant 3D matrix induces $\mathrm{Ca}^{2+}$ oscillations different from the 2D substrate [42]. Therefore, 3D cell-instructive substrate is necessary to better understand the nature of mechanical microenvironment of chondrocytes in the future studies [43]. Our study further confirms the hypothesis that $\mathrm{Ca}^{2+}$ signaling is mediated by the TRPV4 channel, in a stiffness-dependently manner, in chondrocytes sensing substrate stiffness in the isoosmotic (320 mOsm) and hypo-osmotic (180 mOsm) conditions. However, TRPV4-mediated $\mathrm{Ca}^{2+}$ oscillations of chondrocytes exhibited a more complex form during the RVD responding induced by hypoosmotic challenge. For example, soft substrate significantly improved TRPV4-mediated $\mathrm{Ca}^{2+}$ oscillations during the cell swelling, which is consistent with a previous study that soft PCM increases the TRPV4mediated $\mathrm{Ca}^{2+}$ response to hypo-osmotic $(180 \mathrm{mOsm})$ challenge [15]. On the other hand, stiff substrate greatly enhanced the TRPV4-mediated $\mathrm{Ca}^{2+}$ oscillations during the cell recovering. 
Note that substrate stiffness-induced cell stiffness may determine the capacity for cell volume regulation and TRPV4-mediated $\mathrm{Ca}^{2+}$ oscillation. TRPV4 functions as a volume sensor and is activated by increased cell volume irrespective of the molecular mechanism underlying the cell swelling [44]. However, the detailed regulatory mechanisms remain unknown. Therefore, it is needed to further examine the relationship between the rate of membrane deformation and calcium signaling during the cell swelling and recovering progress. Additionally, the $\mathrm{Ca}^{2+}$ oscillation characteristics in native tissue remain to be determined in that it is quite difficult to determine whether the impact of substrate stiffness on $\mathrm{Ca}^{2+}$ signaling is chondroprotective or disease-promoting.

Our work shows that the substrate stiffness plays a critical role in regulating the RVD of chondrocyte and TRPV4-mediated mechanotransduction, though here we do not prove that substrate stiffness directly regulates the TRPV4 function. TRPV4 can be activated by a variety of other stimuli [45]. For example, the cell swelling increases the membrane stretch and leads to increases in contacts between the adhesive molecular and TRPV4, which could activate TRPV4 channels by conformational change-associated "gate" opening through actin reorganization [46]. But it has been proven challenging to demonstrate, in vitro, that the TRPV4 channel is directly gated by mechanical inputs [47].

\section{Conclusions}

In summary, our work highlights the importance of substrate stiffness as a physical cue of the microenvironment in modulating the RVD behavior and TRPV4-mediated $\mathrm{Ca}^{2+}$ signaling of chondrocyte during the hypo-osmotic challenge. Thus, RVD response and TRPV4-mediated $\mathrm{Ca}^{2+}$ signaling, which are critical features of chondrocytes, may change in pathological conditions such as matrix stiffness variance in $\mathrm{OA}$, therefore significantly affecting many cellular functions. For future directions, it is necessary to explore the relationship between calcium signaling and deformation rate in the cell swelling and recovering by direct membrane stretching experiments with different frequency and magnitude. It is well known that a three-dimensional (3D) cell matrix that mimics key factors of tissue is much more representative of the in vivo microenvironment than two-dimensional (2D) substrate. Thus, with an increasing list of available three-dimensional cell-supporting biomaterials, investigating the roles of TRPV4 in regulating chondrocyte RVD and calcium signal based on in vivo animal models would be clinically significant. Our study coupled with a recent progress of ion channels mechanism of cell sensing matrix stiffness is beneficial for the design of cell-instructive scaffolds, which would be crucial for chondrocytes to obtain appropriate mechanobiological behavior. Furthermore, modulation of substrate stiffness-related mechanotransduction pathways, possibly by altering the TRPV4 function, could provide a basis for therapeutic interventions for $\mathrm{OA}$.

\section{Declarations}

\section{Acknowledgements}


This work was supported by research grants (No.11872263 and No.11632013) from the National Natural Science Foundation of China.

\section{Author contributions}

All authors aided in revising this manuscript for intellectual content and approved the final version to be published. Conceived and designed the experiments: QYZ, WYC. Data acquisition: QYZ, MZ, XAW, GLD. Data analysis and interpretation: QYZ, GLD, MZ, XAW, WYC. Wrote and Revised Manuscript: QYZ, MZ, WYC. Critical revision and final approval of the manuscript: All authors.

\section{Competing interest statement}

The authors have no competing interests.

\section{References}

1. R.F. Loeser, S.R. Goldring, C.R. Scanzello, M.B. Goldring, Osteoarthritis: a disease of the joint as an organ, Arthritis Rheum. 64(2012)1697-707.

2. C.A. Poole, Articular cartilage chondrons: form, function and failure, J Anat. 191 (1997)1-13.

3. L.G. Alexopoulos, L.A. Setton, F. Guilak, The biomechanical role of the chondrocyte pericellular matrix in articular cartilage, Acta Biomater. 1(2005)317-25.

4. M. Khoshgoftar, P.A. Torzilli, S.A. Maher, Influence of the pericellular and extracellular matrix structural properties on chondrocyte mechanics, J. Orthop. Res. 36(2018)721-9.

5. M.A. McLeod, R.E. Wilusz, F. Guilak, Depth-dependent anisotropy of the micromechanical properties of the extracellular and pericellular matrices of articular cartilage evaluated via atomic force microscopy, J Biomech. 46(2013)586-92.

6. L.G. Alexopoulos, M.A. Haider, T.P. Vail, F.Guilak, Alterations in the mechanical properties of the human chondrocyte pericellular matrix with osteoarthritis, J. Biomech. Eng. 125(2003)323-33.

7. E.M. Darling, R.E. Wilusz, M.P. Bolognesi, S. Zauscher, F. Guilak, Spatial mapping of the biomechanical properties of the pericellular matrix of articular cartilage measured in situ via atomic force microscopy, Biophys. J. 98(2010)2848-56.

8. R.E. Wilusz, J. Sanchez-Adams, F. Guilak, The structure and function of the pericellular matrix of articular cartilage, Matrix Biol. 39(2014)25-32.

9. F. Guilak, R.J. Nims, A. Dicks, C.L. Wu, I. Meulenbelt. Osteoarthritis as a disease of the cartilage pericellular matrix, Matrix Biol. 71-72(2018):40-50.

10. T.L. Vincent, Targeting mechanotransduction pathways in osteoarthritis: a focus on the pericellular matrix, Curr Opin Pharmacol. 13(2013)449 - 54.

11. M. Danalache, R. Kleinert, J. Schneider, A.L Erler, M. Schwitalle, R. Riester, F. Traub, U.K. Hofmann, Changes in stiffness and biochemical composition of the pericellular matrix as a function of spatial chondrocyte organisation in osteoarthritic cartilage, Osteoarthritis Cartilage. 27(2019)823 - 32. 
12. P. Tanska, S.M. Turunen, S.K. Han, P. Julkunen, W. Herzog, R.K. Korhonen, Superficial collagen fibril modulus and pericellular fixed charge density modulate chondrocyte volumetric behavior in early osteoarthritis, Comput Math Methods Med. (2013)164146.

13. N.A. Zelenski, H.A. Leddy, J. Sanchez-Adams, J. Zhang, P. Bonaldo, W. Liedtke, F. Guilak, Type VI collagen regulates pericellular matrix properties, chondrocyte swelling, and mechanotransduction in mouse articular cartilage, Arthritis Rheum. 67(2015)1286-94.

14. P.G. Bush, A.C. Hall, Regulatory volume decrease (RVD) by isolated and in situ bovine articular chondrocytes. J Cell Physiol.187(2001)304-14.

15. V. Vogel, Unraveling the Mechanobiology of Extracellular Matrix, Annu Rev Physiol. 80(2018)353 87.

16. B. Cao, R. Peng, Z. Li, J. Ding, Effects of spreading areas and aspect ratios of single cells on dedifferentiation of chondrocytes, Biomaterials. 35(2014) 6871-81.

17. Q.Y. Zhang, Y. Yu, H.C. Zhao, The effect of matrix stiffness on biomechanical properties of chondrocytes, Acta Biochim Biophys Sin. 48(2016)958-65.

18. P.G. Bush, A.C. Hall, The volume and morphology of chondrocytes within non-degenerate and degenerate human articular cartilage, Osteoarthritis Cartilage. 11(2003)242-51.

19. P.G. Bush, A.C. Hall, Passive osmotic properties of in situ human articular chondrocytes within nondegenerate and degenerate cartilage, J Cell Physiol. 204(2005)309-19.

20. A.C. Hall, The role of chondrocyte morphology and volume in controlling phenotype-implications for osteoarthritis, cartilage repair, and cartilage engineering, Curr Rheumatol Rep. 21(2019) 38.

21. R. Lewis, C.H. Feetham, R. Barrett-Jolley, Cell volume regulation in chondrocytes, Cell Physiol Biochem. 28(2011)1111-22.

22. F. Guilak, H.A. Leddy, W. Liedtke, Transient receptor potential vanilloid 4: the sixth sense of the musculoskeletal system?, Ann N Y Acad Sci. 1192(2010)404-9.

23. M.N. Phan, H.A. Leddy, B.J. Votta, S. Kumar, D.S. Levy, D.B. Lipshutz, S.H. Lee, W. Liedtke, F. Guilak, Functional characterization of TRPV4 as an osmotically sensitive ion channel in porcine articular chondrocytes, Arthritis Rheum. 60(2009)3028-37.

24. M.R. Servin-Vences, J. Richardson, G.R. Lewin, K. Poole, Mechanoelectrical transduction in chondrocytes, Clin Exp Pharmacol Physiol. 45(2018) 481-8.

25. C. Goswami, J. Kuhn, P.A. Heppenstall, T. Hucho, Importance of non-selective cation channel TRPV4 interaction with cytoskeleton and their reciprocal regulations in cultured cells, PLoS One. 5(2010) e11654.

26. B.D. Matthews, C.K. Thodeti, J.D. Tytell, A. Mammoto, D.R. Overby, D.E. Ingber, Ultra-rapid activation of TRPV4 ion channels by mechanical forces applied to cell surface beta1 integrins, Integr Biol. 2(2010)435-42.

27. C.J. O’Conor, H.A. Leddy, H.C. Benefield, W.B. Liedtke, F. Guilak, TRPV4-mediated mechanotransduction regulates the metabolic response of chondrocytes to dynamic loading, Proc 
Natl Acad Sci U S A. 111(2014)1316-21.

28. T. Kobayashi, M. Sokabe, Sensing substrate rigidity by mechanosensitive ion channels with stress fibers and focal adhesions, Curr Opin Cell Biol. 22(2010)669-76.

29. C.L. Jablonski, S. Ferguson, A. Pozzi, A.L. Clark, Integrin a1ß1 participates in chondrocyte transduction of osmotic stress, Biochem Biophys Res Commun. 445(2014)184-90.

30. D.E. Clapham, Calcium signaling, Cell. 131(2007)1047-58.

31. X. Gong, W. Xie, B. Wang, L. Gu, F. Wang, X. Ren, C. Chen, L. Yang, Altered spontaneous calcium signaling of in situ chondrocytes in human osteoarthritic cartilage, Sci Rep. 7(2017)17093.

32. Q.Y. Zhang, Y.Y. Zhang, J. Xie, C.X. Li, W.Y. Chen, B.L. Liu, et al, Stiff substrates enhance cultured neuronal network activity, Scientific Reports. 4(2014)6215.

33. M. Gosset, F. Berenbaum, S. Thirion, C. Jacques, Primary culture and phenotyping of murine chondrocytes, Nat Protoc. 3(2008)1253-60.

34. Z. Wang, J. Irianto, S. Kazun, W. Wang, M.M. Knight, The rate of hypo-osmotic challenge influences regulatory volume decrease (RVD) and mechanical properties of articular chondrocytes, Osteoarthritis Cartilage. 23(2015)289-99.

35. E.M. Darling, S. Zauscher, J.A. Block, F. Guilak, A thin-layer model for viscoelastic, stress-relaxation testing of cells using atomic force microscopy: do cell properties reflect metastatic potential?, Biophys. J. 92(2007)1784-91.

36. S.W Donahue, H.J Donahue, C.R Jacobs, Osteoblastic cells have refractory periods for fluid-flowinduced intracellular calcium oscillations for short bouts of flow and display multiple low-magnitude oscillations during long-term flow, Biomech. J. 36(2003)35-43.

37. J. Irianto, J. Swift, R.P. Martins, G.D. McPhail, M.M. Knight, D.E. Discher, D.A. Lee, Osmotic challenge drives rapid and reversible chromatin condensation in chondrocytes, Biophys. J. 104(2013)759-69.

38. P.A. Janmey, D.A. Fletcher, C.A. Reinhart-King, Stiffness sensing by cells, Physiol. Rev. 100(2020)695-724.

39. F. Guilak, G.R. Erickson, H.P. Ting-Beall, The effects of osmotic stress on the viscoelastic and physical properties of articular chondrocytes, Biophys. J. 82(2002)720-7.

40. M. Guo, A.F. Pegoraro, A. Mao, E.H. Zhou, P.R. Arany, Y. Han, et al, Cell volume change through water efflux impacts cell stiffness and stem cell fate. Proc Natl Acad Sci U S A. 114(2017) E8618-27.

41. Y. Zhou, M. Park, E. Cheung, L. Wang, X.L. Lu, The effect of chemically defined medium on spontaneous calcium signaling of in situ chondrocytes during long-term culture, Biomech. J. 48(2015) 990-6.

42. Q.Y. Zhang, J.D. Bai, X.A. Wu, X.N. Liu, M. Zhang, W.Y. Chen, Microniche geometry modulates the mechanical properties and calcium signaling of chondrocytes, Biomech.J. 104 (2020) 109729.

43. K.E. Scott, K. Rychel, S. Ranamukhaarachchi, P. Rangamani, S.I. Fraley, Emerging themes and unifying concepts underlying cell behavior regulation by the pericellular space, Acta Biomater. 96(2019)81-98. 
44. T.L. Toft-Bertelsen, O. Yarishkin, S. Redmon, T.T.T. Phuong, D. Križaj, N. MacAulay, Volume sensing in the transient receptor potential vanilloid 4 ion channel is cell type-specific and mediated by an Nterminal volume-sensing domain, J. Biol. Chem. 294(2019)18421-34.

45. W.G. Darby, M.S. Grace, S. Baratchi, P. Mclntyre, Modulation of TRPV4 by diverse mechanisms, Int J Biochem Cell Biol. 78(2016)217-28.

46. M. Jin, J. Berrout, R.G. O'Neil, Regulation of TRP Channels by Osmomechanical Stress. In: M.X. Zhu, editor. TRP Channels. Boca Raton (FL): CRC Press/Taylor \& Francis. Chapter 16(2011).

47. A.P. Christensen, D.P. Corey, TRP channels in mechanosensation: direct or indirect activation?, Nat Rev Neurosci. 8(2007)510-21.

\section{Tables}

Due to technical limitations, table 1 is only available as a download in the Supplemental Files section.

\section{Figures}


A

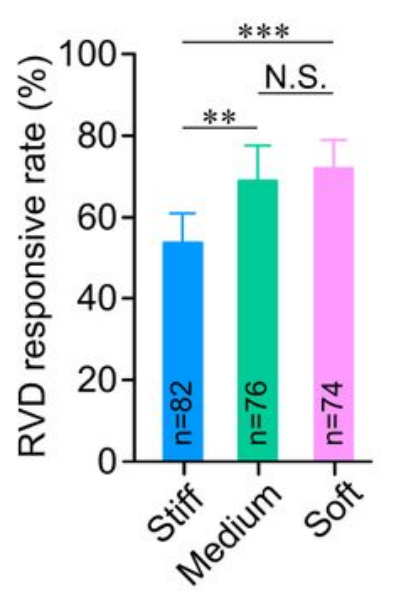

C

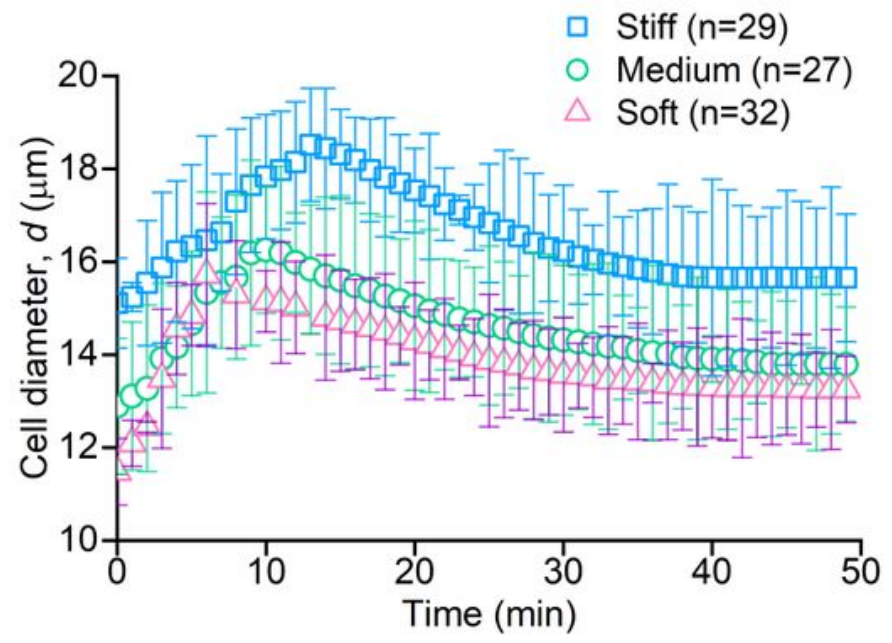

E

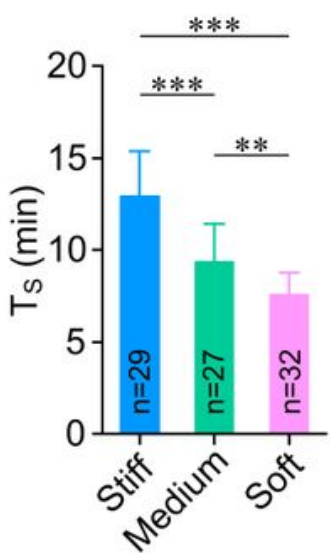

$\mathbf{F}$

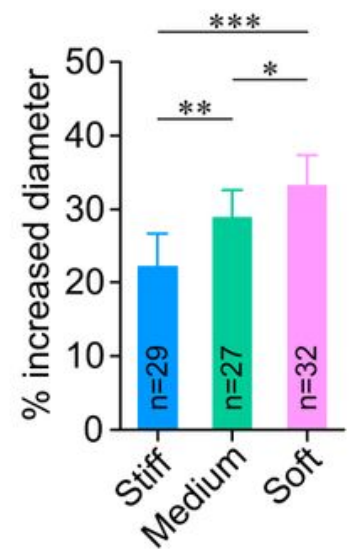

B

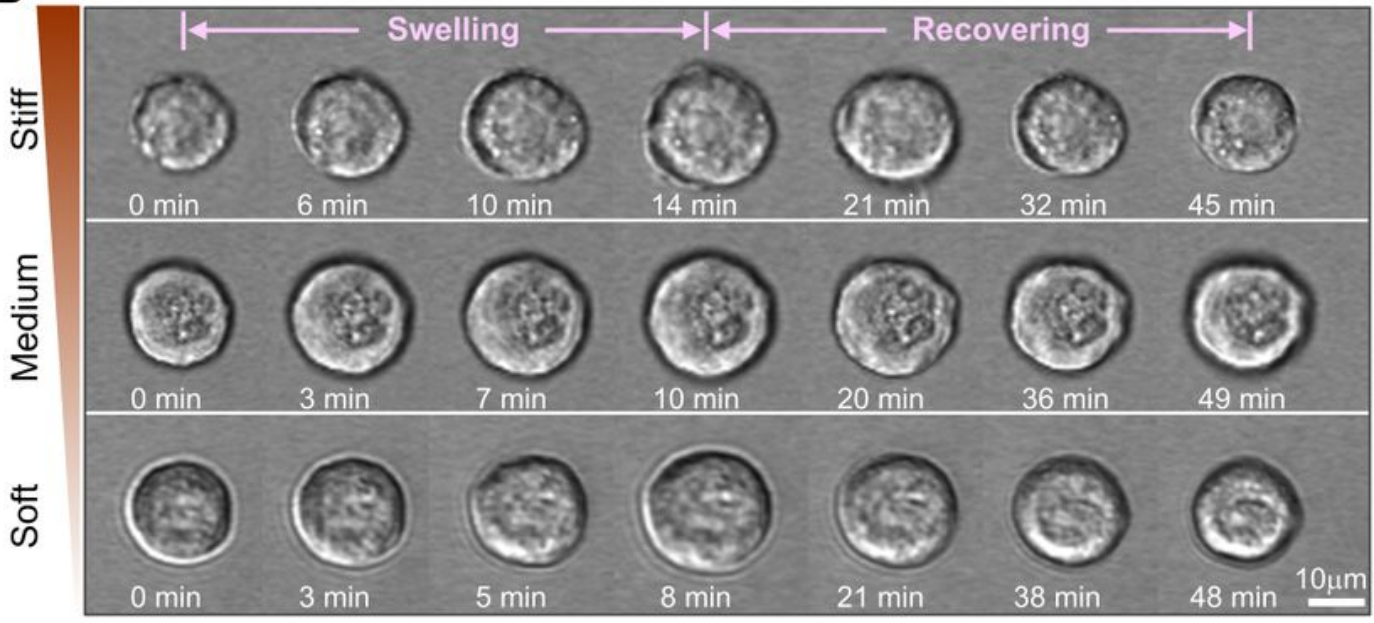

D

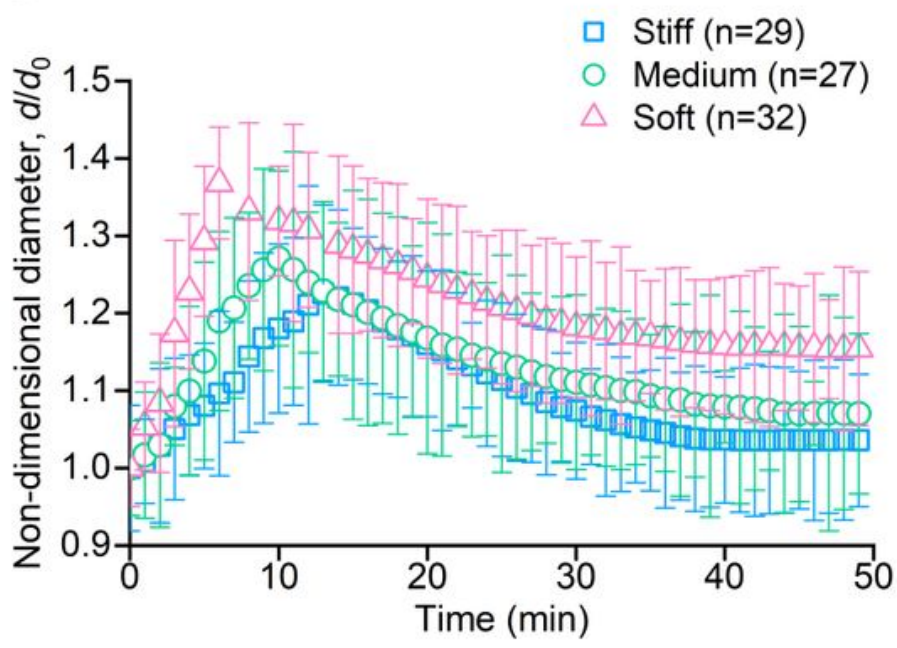

G

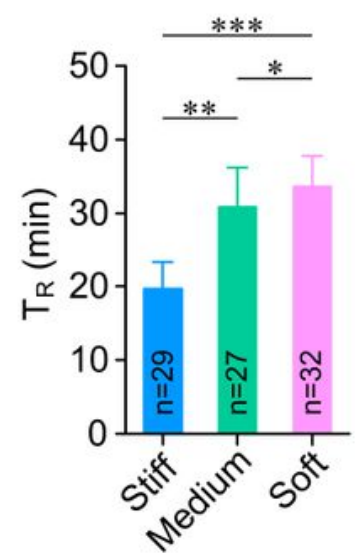

H

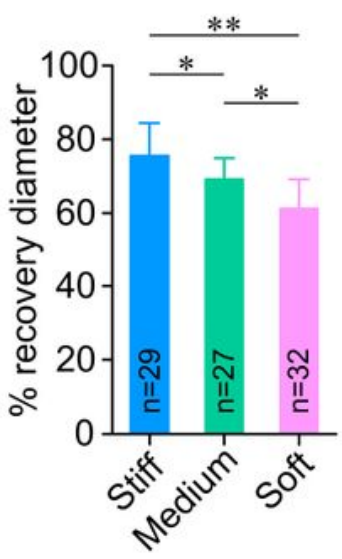

I

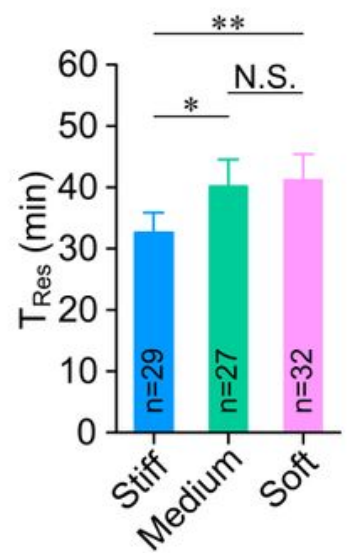

\section{Figure 1}

Substrate stiffness affects the hypo-osmotic challenge (180 mOsm)-induced RVD response of chondrocytes. (A) The percentage of RVD response in chondrocytes on stiff substrate $(55 \pm 6 \%)$ was significantly lower than that on medium $(68 \pm 6 \%)$ and softy substrate $(72 \pm 8 \%)$, re-spectively. The analysis of RVD responsive rate in 82 chondrocytes on the stiff substrate, 76 chondrocytes on the medium substrate and 74 chondrocytes on soft substrate. Each group is from 10 different experiments. 
(B) Representative time elapse recording of the RVD response in three chondrocytes from the stiff, medium and soft substrate, respectively. (C) The diameter (d) changes of chondrocytes on variable stiffness substrates during the complete RVD response. (D) The non-dimensional diameter $(\mathrm{d} / \mathrm{d} 0)$ change of chondrocytes on variable stiffness substrates during the RVD response. The trend of $d / d 0$ change with time is consistent with Fig.1C. (E) The stiff substrate significantly increased the mean swelling time (TS) of chondrocytes during the cell swelling. $(F)$ The percentage increase of chondrocyte diameter was increased significantly with substrate stiffness decreasing during the cell swelling. (G) The mean recovery time (TR) of chondrocyte on the softer substrate was dramatically prolonged during the cell recovering. $(\mathrm{H})$ The percentage of chondrocyte recovery diameter was increased with substrate stiffness increas-ing. (I) The mean RVD responding time (TRes) of chondrocyte on the soft substrate was signifi-cantly longer than that on stiff substrate. The swelling and recovering processes of chondrocytes significantly depend on the substrate stiffness during RVD response. Bars represent mean with S.D. $n$ value for panels A,C-F denote the numbers of chondrocytes analyzed from six different experiments per substrate stiffness. Statistical significance was tested with one-way ANOVA and post hoc Tukey's test. N.S., not significant $(p>0.05) .{ }^{*}, p<0.05 ; * \star, p<0.01 ; * \star \star, p<0.001$. 
A

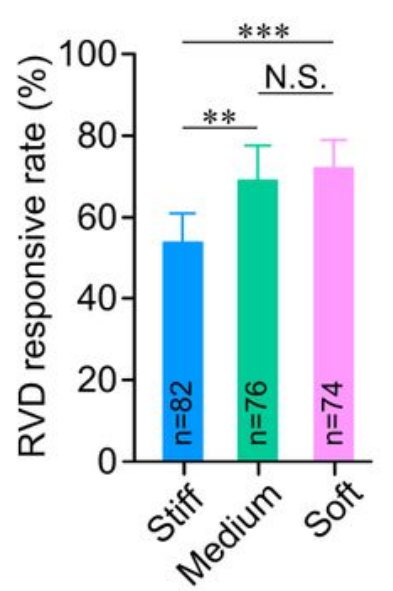

C

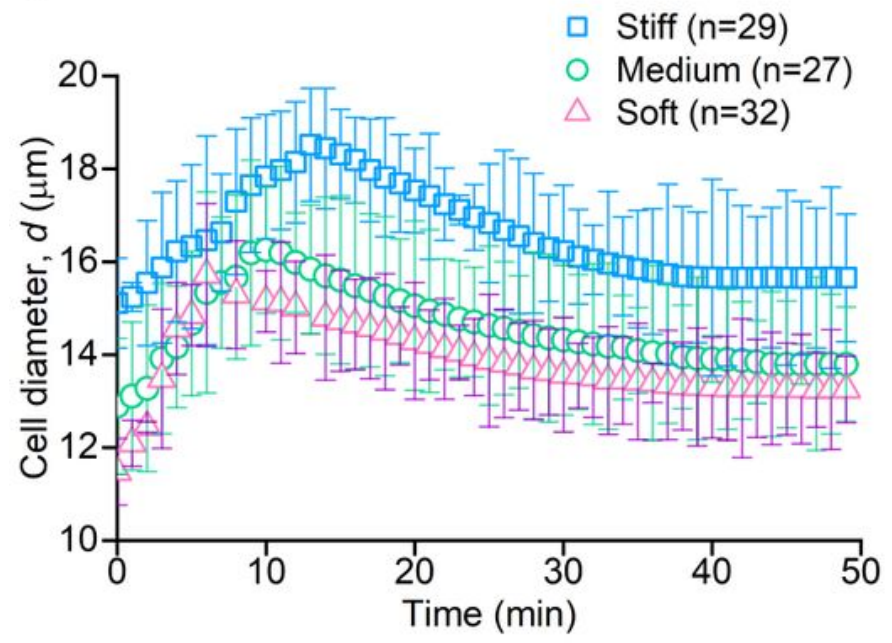

E

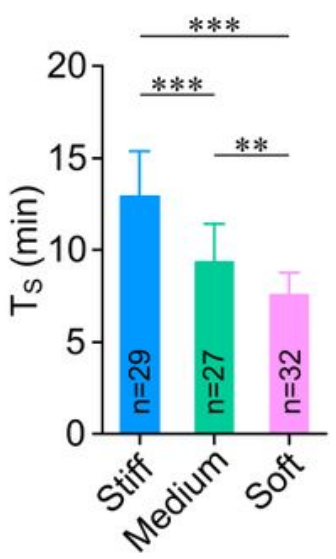

$\mathbf{F}$

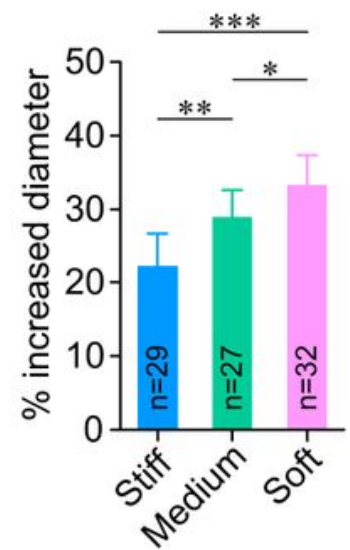

B

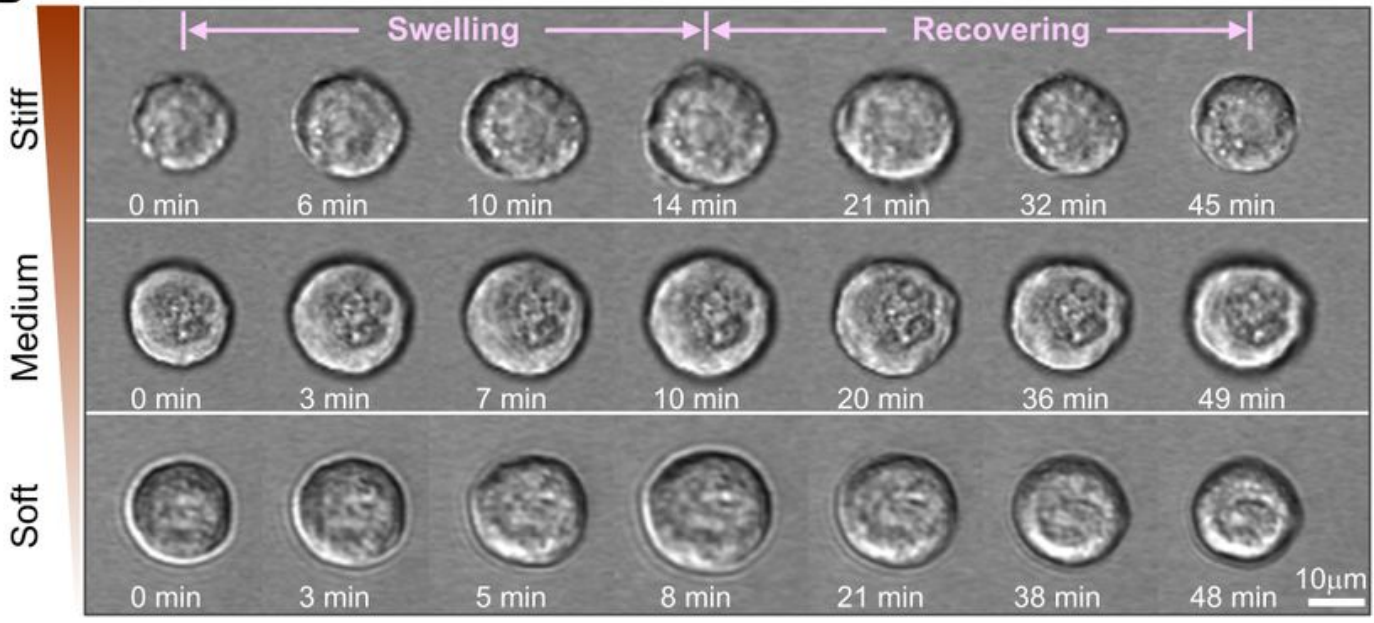

D

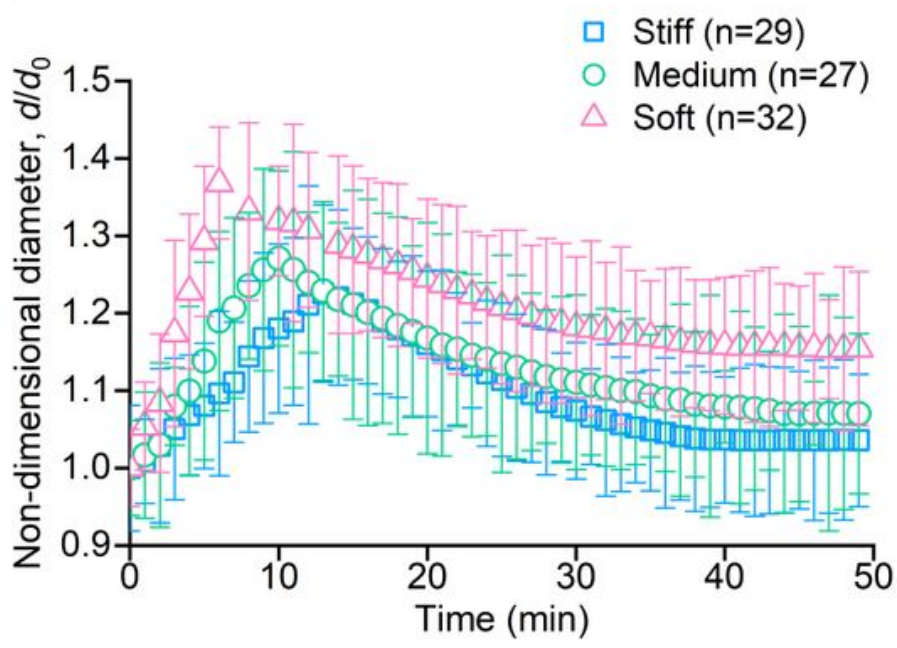

G

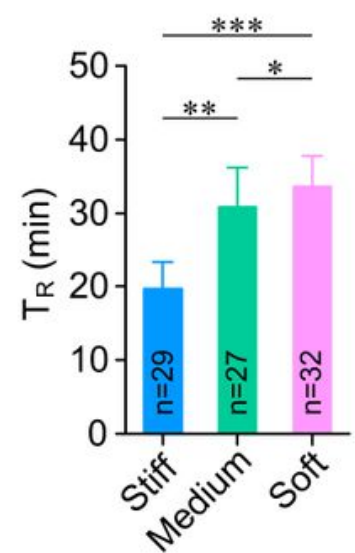

H

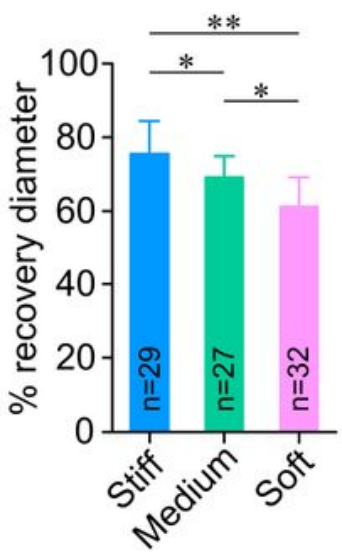

I

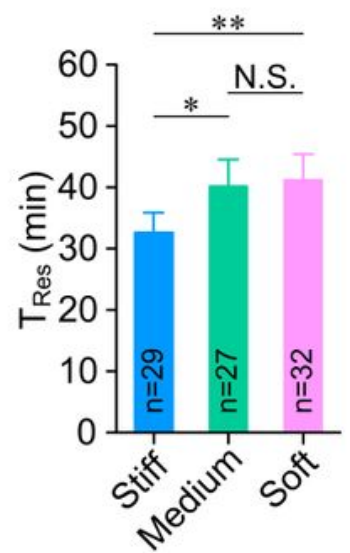

\section{Figure 1}

Substrate stiffness affects the hypo-osmotic challenge (180 mOsm)-induced RVD response of chondrocytes. (A) The percentage of RVD response in chondrocytes on stiff substrate $(55 \pm 6 \%)$ was significantly lower than that on medium $(68 \pm 6 \%)$ and softy substrate $(72 \pm 8 \%)$, re-spectively. The analysis of RVD responsive rate in 82 chondrocytes on the stiff substrate, 76 chondrocytes on the medium substrate and 74 chondrocytes on soft substrate. Each group is from 10 different experiments. 
(B) Representative time elapse recording of the RVD response in three chondrocytes from the stiff, medium and soft substrate, respectively. (C) The diameter (d) changes of chondrocytes on variable stiffness substrates during the complete RVD response. (D) The non-dimensional diameter $(\mathrm{d} / \mathrm{d} 0)$ change of chondrocytes on variable stiffness substrates during the RVD response. The trend of $d / d 0$ change with time is consistent with Fig.1C. (E) The stiff substrate significantly increased the mean swelling time (TS) of chondrocytes during the cell swelling. $(F)$ The percentage increase of chondrocyte diameter was increased significantly with substrate stiffness decreasing during the cell swelling. (G) The mean recovery time (TR) of chondrocyte on the softer substrate was dramatically prolonged during the cell recovering. $(\mathrm{H})$ The percentage of chondrocyte recovery diameter was increased with substrate stiffness increas-ing. (I) The mean RVD responding time (TRes) of chondrocyte on the soft substrate was signifi-cantly longer than that on stiff substrate. The swelling and recovering processes of chondrocytes significantly depend on the substrate stiffness during RVD response. Bars represent mean with S.D. $n$ value for panels A,C-F denote the numbers of chondrocytes analyzed from six different experiments per substrate stiffness. Statistical significance was tested with one-way ANOVA and post hoc Tukey's test. N.S., not significant $(p>0.05) .{ }^{*}, p<0.05 ; * *, p<0.01 ; * * *, p<0.001$.

A

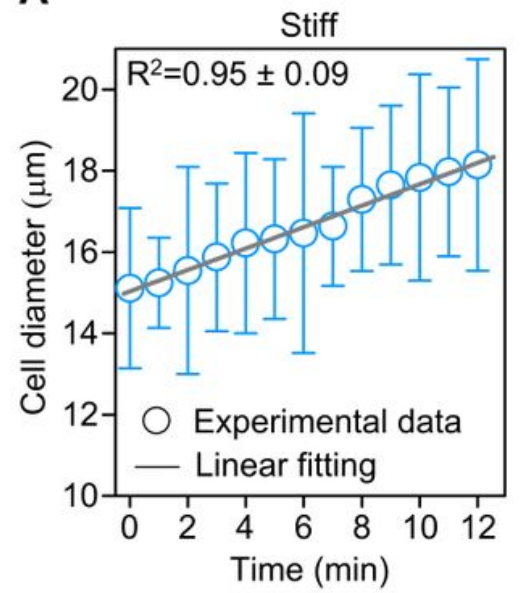

Time (min)
B

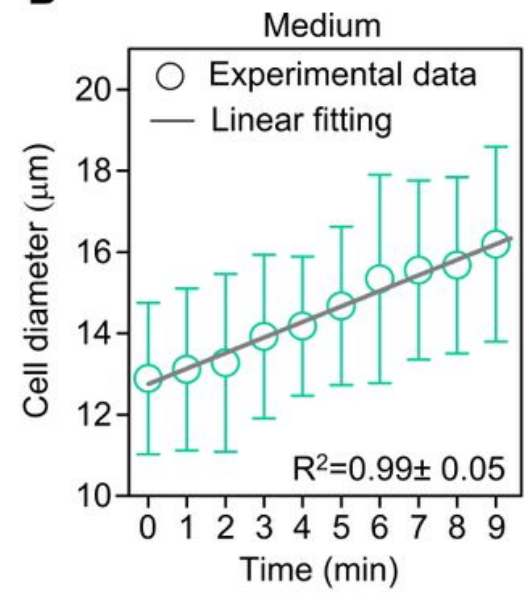

C

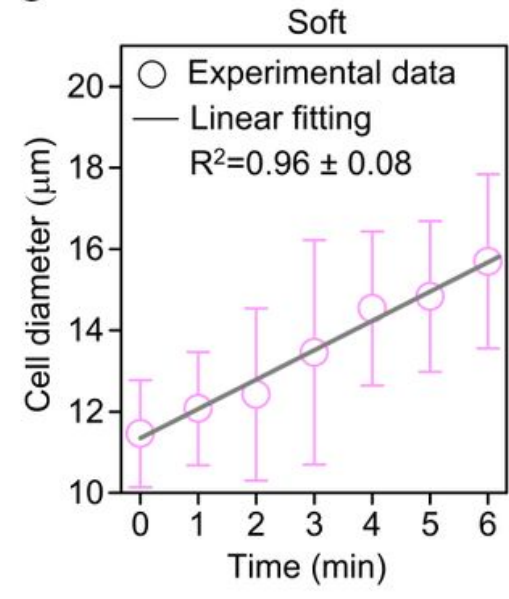

D

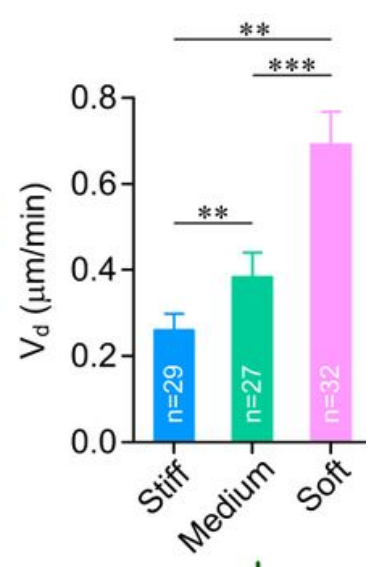

Cell swelling

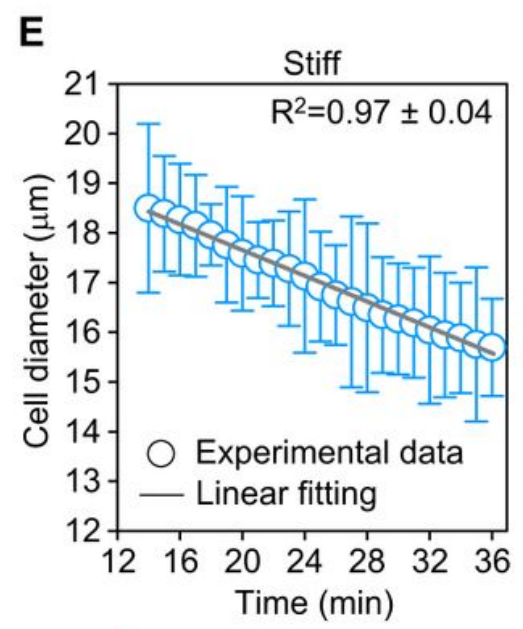

F

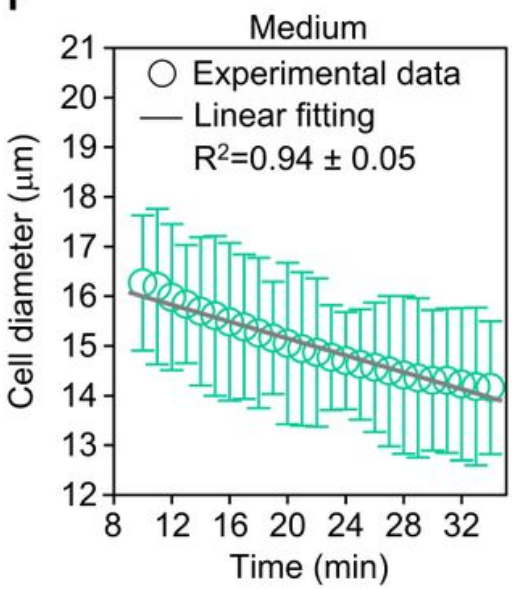

G

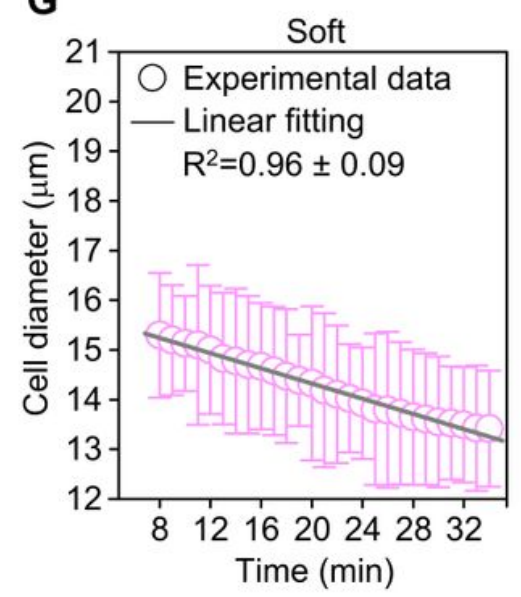

H

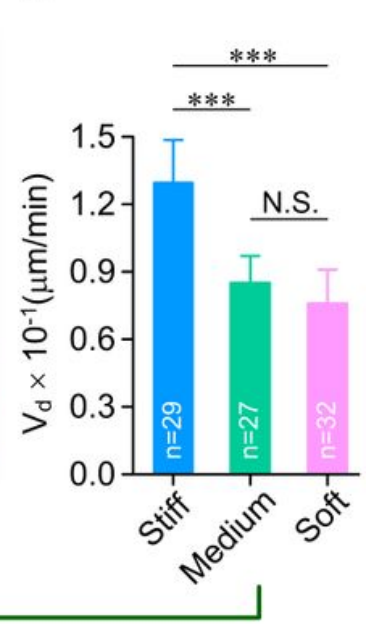

Figure 2

Cell recovering 
Quantification of chondrocyte diameter rate during the hypo-osmotic challenge (180 mOsm)-induced RVD response. (A), (B) and (C) are the linearly fitting of cell diameter vs time curves on variable stiffness substrates during the cell swelling. The diameter rate, $\mathrm{Vd}$ is the slope of a line. The cell diameter rate in stiff, medium and soft substrate are $\mathrm{Vd}=0.26 \pm 0.02 \mu \mathrm{m} / \mathrm{min}, \mathrm{Vd}=0.38 \pm 0.03 \mu \mathrm{m} / \mathrm{min}$ and $\mathrm{Vd}=0.72 \pm 0.04$ $\mu \mathrm{m} / \mathrm{min}$, respectively. (D) Soft substrate significant-ly increased the cell diameter rate during the cell swelling. (E), (F) and (G) are the linearly fitting of cell diameter vs time curves on substrates of varying stiffness during the cell recovering. The diameter change curve from the maximum diameter to the state of equilibrium was used to line-arly fit. The cell diameter change rate in stiff, medium and soft substrate are $V d=0.13 \pm 0.02 \mu \mathrm{m} / \mathrm{min}, V d=0.09 \pm 0.01 \mu \mathrm{m} / \mathrm{min}$ and $V d=0.08 \pm 0.01 \mu \mathrm{m} / \mathrm{min}$, respectively. $(H)$ Stiff substrate enhanced the diameter rate of chondrocytes during the cell recovering process. A higher coefficient of determination (R2) indicates a better linear match. $n$ value for panels $D$ and $H$ denote the numbers of chondrocytes analyzed from six different experiments per substrate stiffness. Bars represent mean with S.D. Statistical significance was tested with one-way ANOVA and post hoc Tukey's test. N.S., not significant $(p>0.05) . * *, p<0.01 ; * \star *, p<0.001$

A
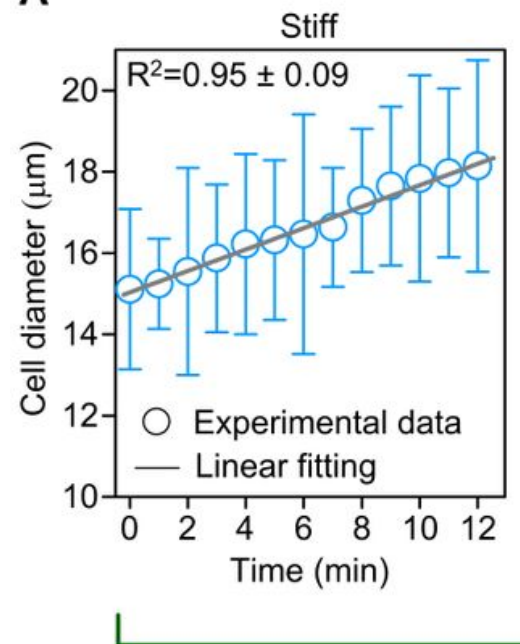

E

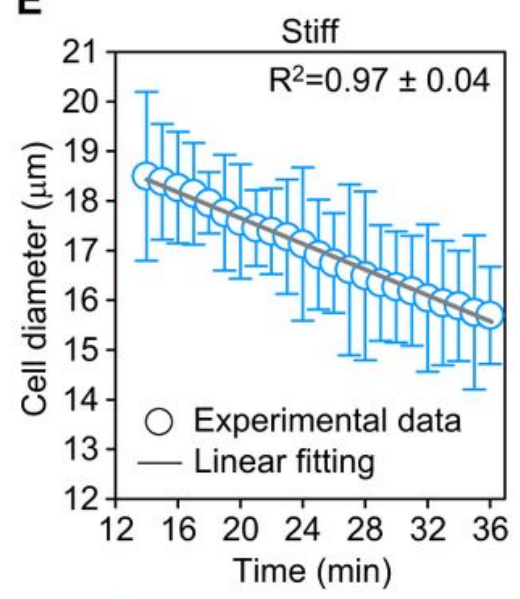

B

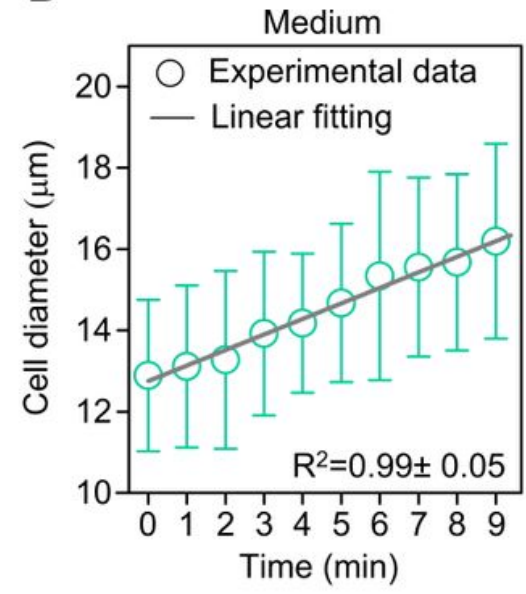

C

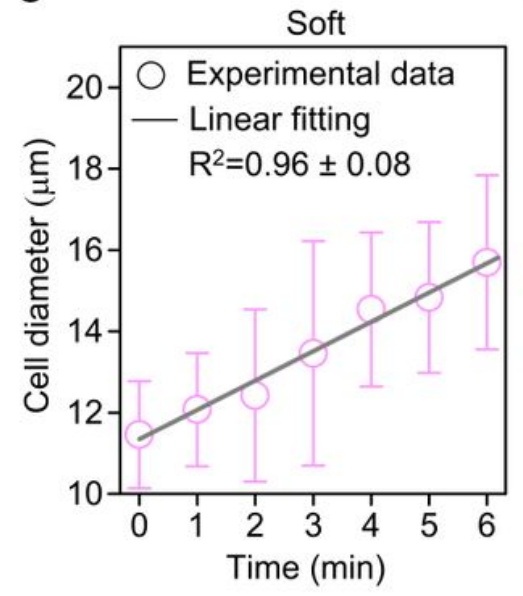

D

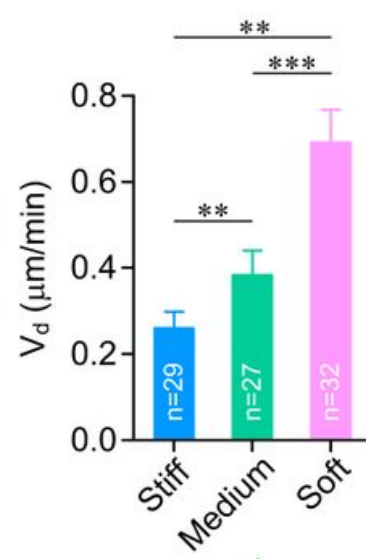

Cell swelling

F

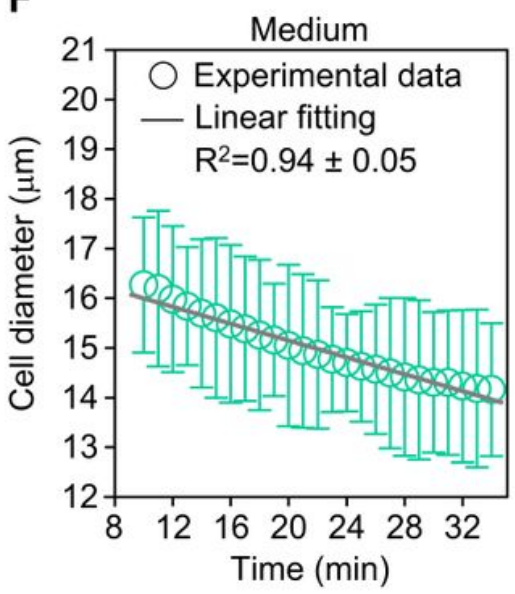

G

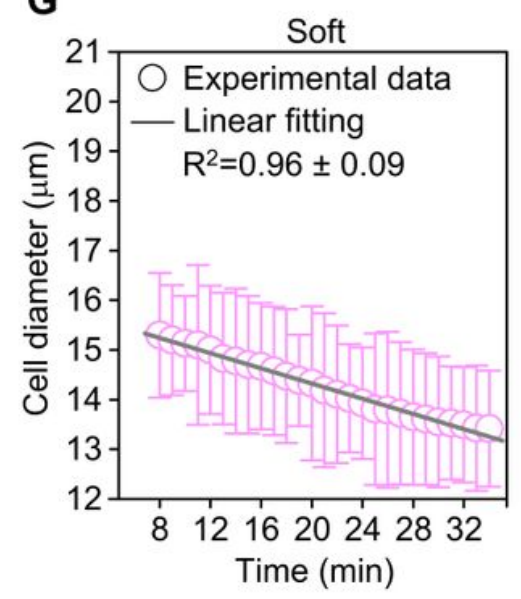

H

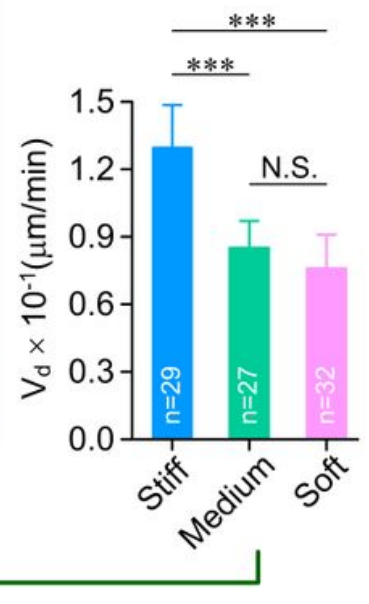

Figure 2 
Quantification of chondrocyte diameter rate during the hypo-osmotic challenge (180 mOsm)-induced RVD response. (A), (B) and (C) are the linearly fitting of cell diameter vs time curves on variable stiffness substrates during the cell swelling. The diameter rate, $\mathrm{Vd}$ is the slope of a line. The cell diameter rate in stiff, medium and soft substrate are $V d=0.26 \pm 0.02 \mu \mathrm{m} / \mathrm{min}, \mathrm{Vd}=0.38 \pm 0.03 \mu \mathrm{m} / \mathrm{min}$ and $\mathrm{Vd}=0.72 \pm 0.04$ $\mu \mathrm{m} / \mathrm{min}$, respectively. (D) Soft substrate significant-ly increased the cell diameter rate during the cell swelling. (E), (F) and (G) are the linearly fitting of cell diameter vs time curves on substrates of varying stiffness during the cell recovering. The diameter change curve from the maximum diameter to the state of equilibrium was used to line-arly fit. The cell diameter change rate in stiff, medium and soft substrate are $V d=0.13 \pm 0.02 \mu \mathrm{m} / \mathrm{min}, V d=0.09 \pm 0.01 \mu \mathrm{m} / \mathrm{min}$ and $V d=0.08 \pm 0.01 \mu \mathrm{m} / \mathrm{min}$, respectively. $(H)$ Stiff substrate enhanced the diameter rate of chondrocytes during the cell recovering process. A higher coefficient of determination (R2) indicates a better linear match. $n$ value for panels $D$ and $H$ denote the numbers of chondrocytes analyzed from six different experiments per substrate stiffness. Bars represent mean with S.D. Statistical significance was tested with one-way ANOVA and post hoc Tukey's test. N.S., not significant $(p>0.05) . * \star, p<0.01 ; * \star \star, p<0.001$ 
A

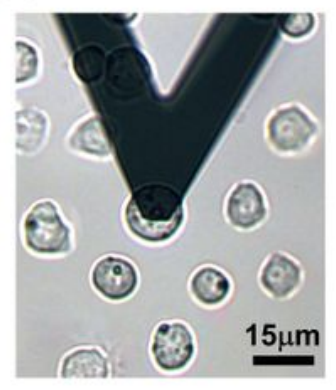

D

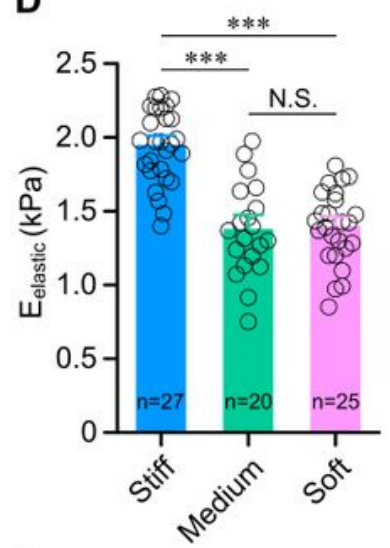

H

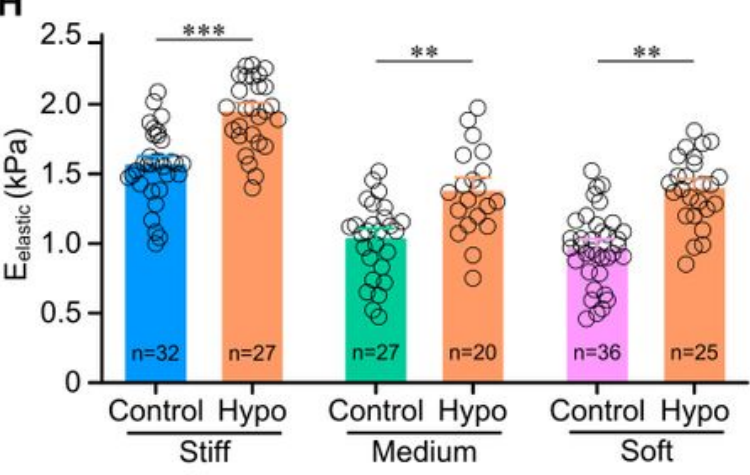

J

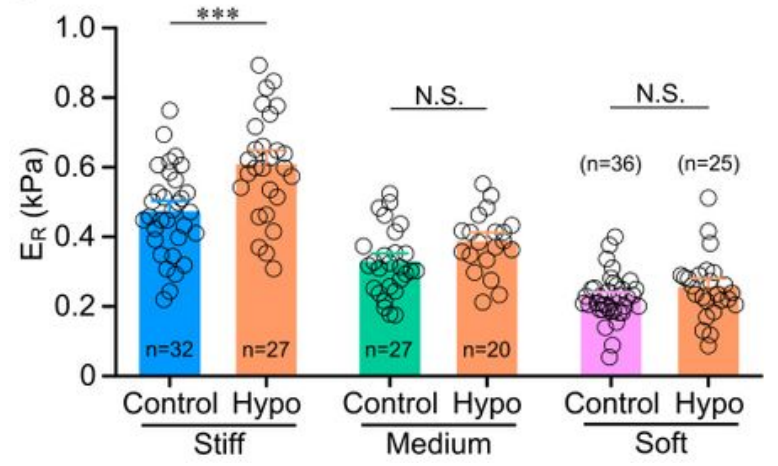

B

E
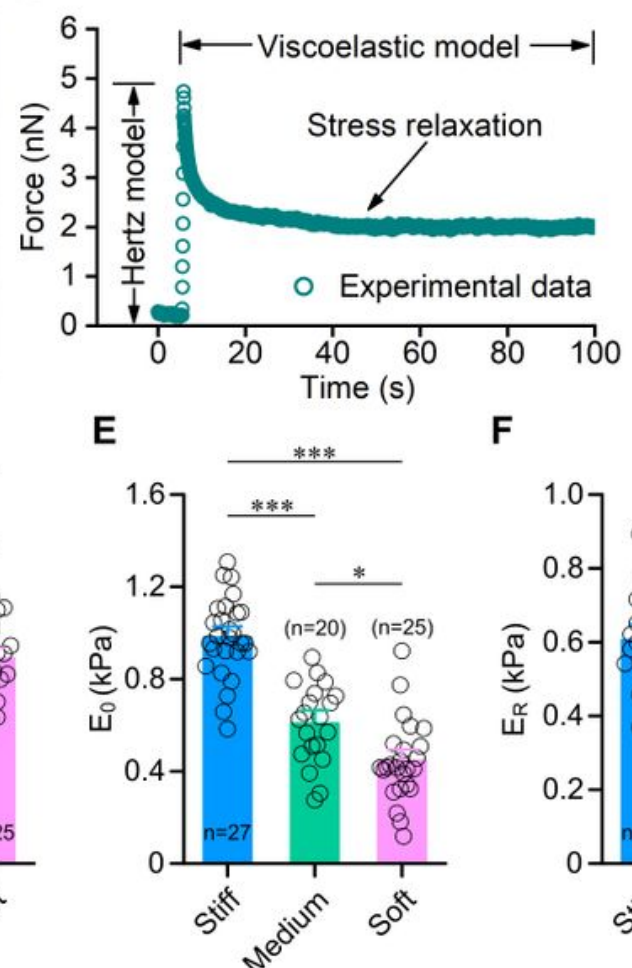

F

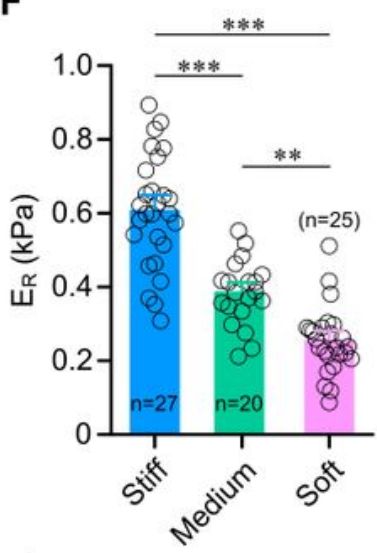

G

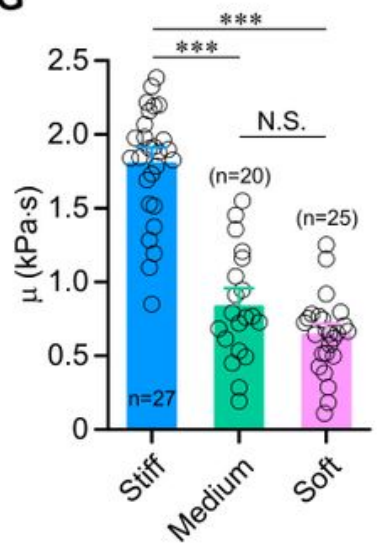

I

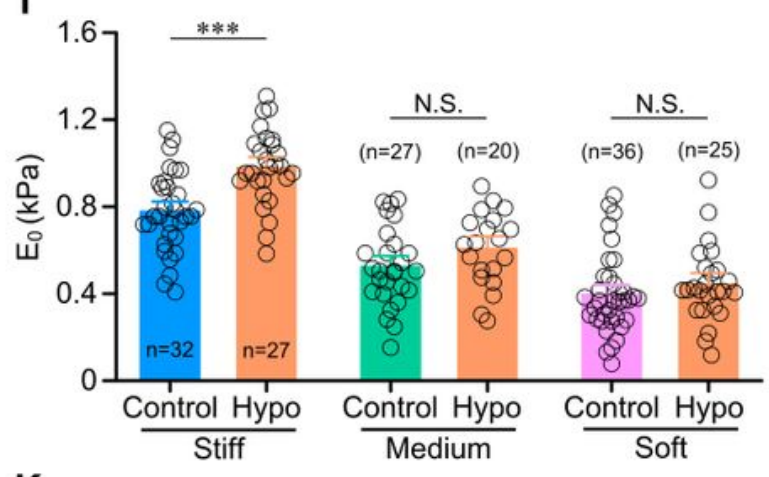

K

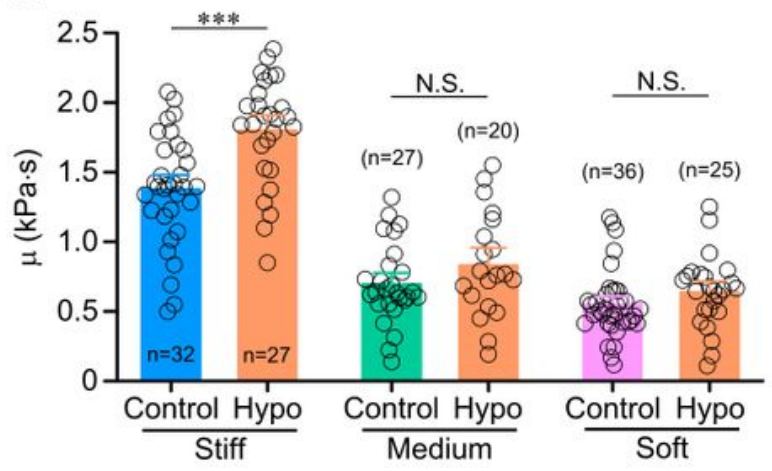

Figure 3

AFM indentation and the analysis of mechanical properties of chondrocyte on variable stiffness substrates during the cell swelling in 180 mOsm medium. All cells were seeded at density of $2 \times 104$ cells/cm2 in the AFM experiment. (A) Schematic illustration of the AFM measurement. Chondrocytes maintained a spherical shape after 24 hours of cell seeding onto variable stiffness substrates. The AFM probe was positioned over the perinuclear region of single chondrocyte in AFM experiments. Only 
chondrocytes with the RVD response were chosen for mechanical anal-ysis. AFM experiments were performed $3 \mathrm{~min}$ after exposure to the hypo-osmotic challenge (180 mOsm). (B) Representative elastic and time-dependent stress relaxation response of single chondrocyte in AFM experiments. Chondrocyte usually exhibits the typical elastic response and subsequently stress-relaxation process. The Eelastic of a chondrocyte was extracted from the initial indentation according to the Hertz model. The viscoelastic properties were extracted by fitting the stress-relaxation curve. (C) Representative stress-relaxation curves and theoretical fitting of chondrocytes from variable stiffness substrates during the cell swelling, respectively. A higher coefficient of determination (R2) indicates a better match between the stress relaxation model and experimental data. (D-G) Comparison of mechanical parameters of chondrocytes on varia-ble stiffness substrates during the cell swelling. Stiff substrate markedly increased the mechanical parameters (Eelastic, ER, EO and $\mu$ ) of chondrocytes. (H-K) Comparison of mechanical pa-rameters of chondrocytes on variable stiffness substrates in the iso-osmotic ( $320 \mathrm{mOsm}$ ) (control group) and hypoosmotic (180 mOsm) medium. N.S., not significant ( $p>0.05) .{ }^{*}, p<0.05 ; * \star, p<0.01 ; * \star \star, p<0.001$; one-way ANOVA and post hoc Tukey's test. In each panel, bar height denotes the mean of the data points, error bars denote standard error of the mean; the number of cells analyzed from variable stiffness substrates is specified above each bar. 
A

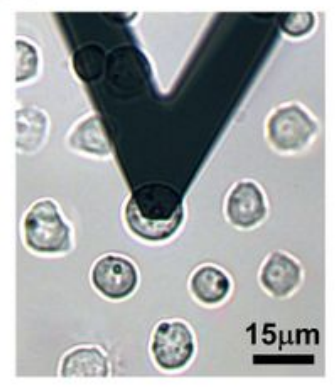

D

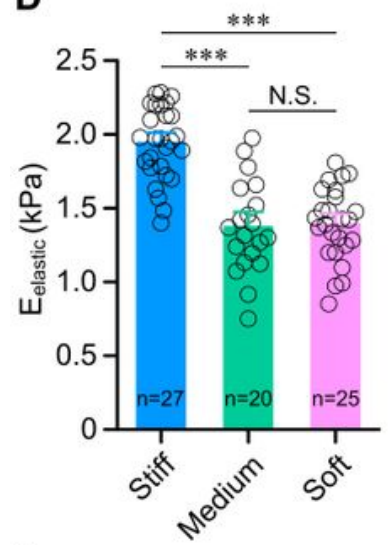

H

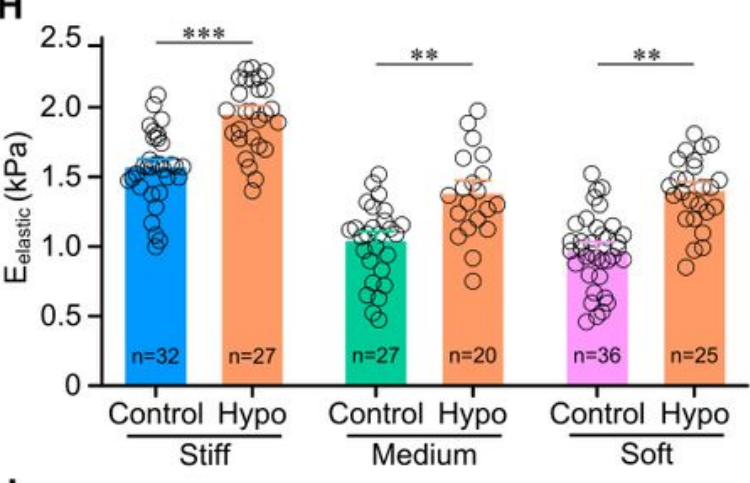

J

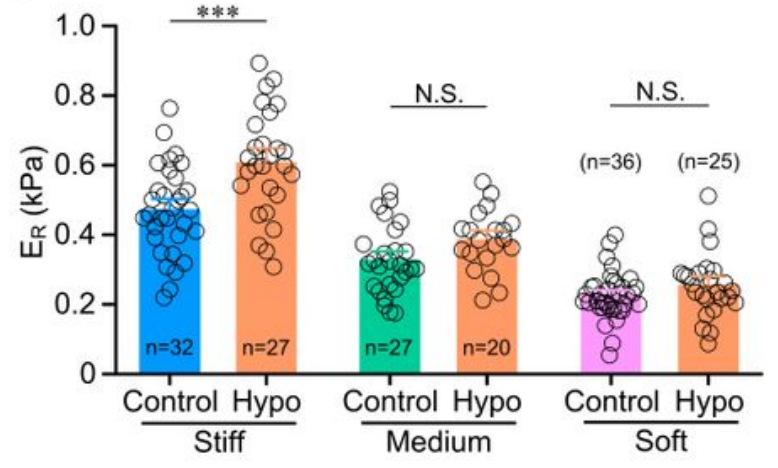

B

E
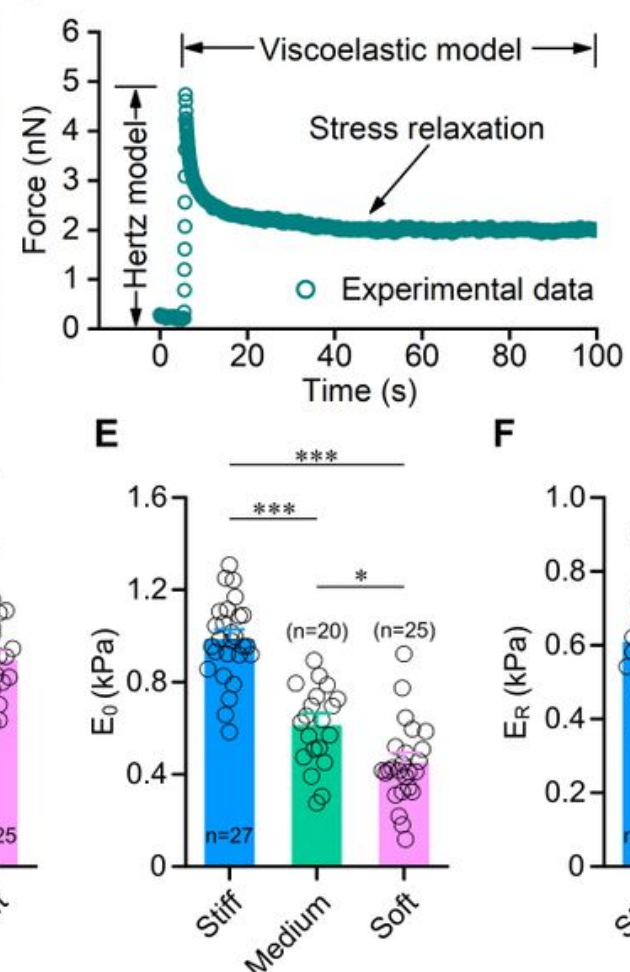

F

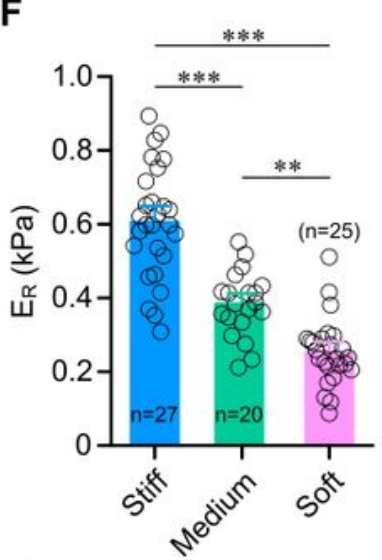

G

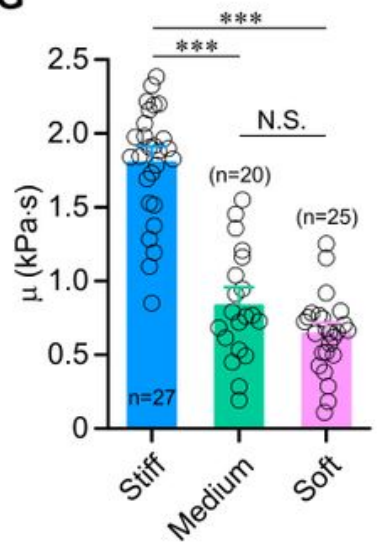

I

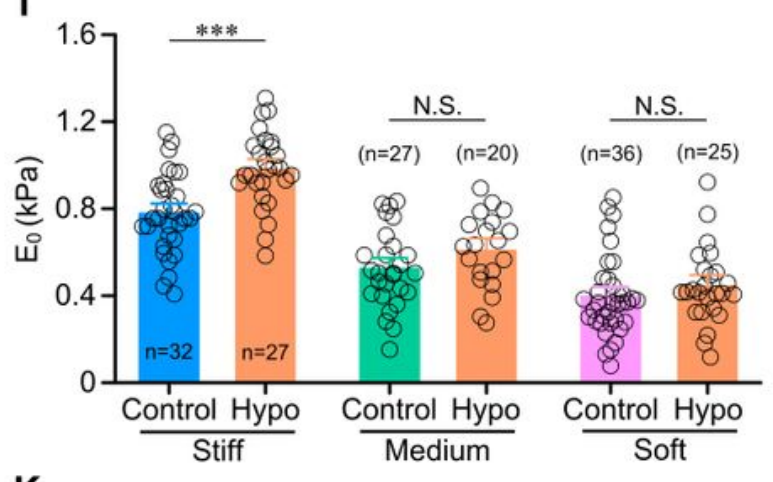

K

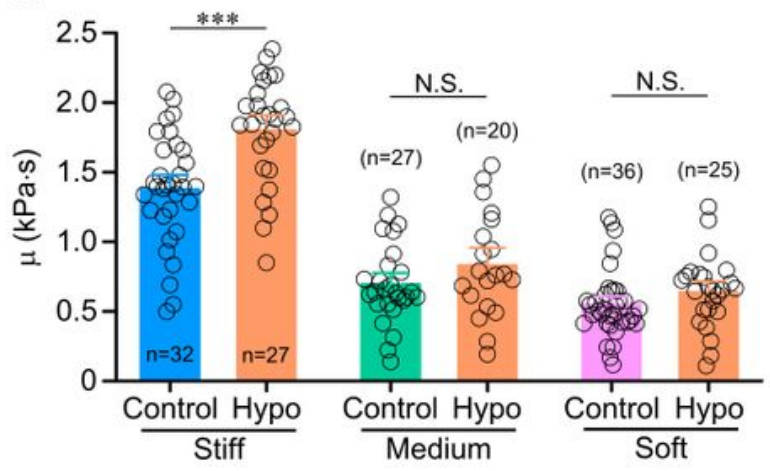

Figure 3

AFM indentation and the analysis of mechanical properties of chondrocyte on variable stiffness substrates during the cell swelling in 180 mOsm medium. All cells were seeded at density of $2 \times 104$ cells/cm2 in the AFM experiment. (A) Schematic illustration of the AFM measurement. Chondrocytes maintained a spherical shape after 24 hours of cell seeding onto variable stiffness substrates. The AFM probe was positioned over the perinuclear region of single chondrocyte in AFM experiments. Only 
chondrocytes with the RVD response were chosen for mechanical anal-ysis. AFM experiments were performed $3 \mathrm{~min}$ after exposure to the hypo-osmotic challenge (180 mOsm). (B) Representative elastic and time-dependent stress relaxation response of single chondrocyte in AFM experiments. Chondrocyte usually exhibits the typical elastic response and subsequently stress-relaxation process. The Eelastic of a chondrocyte was extracted from the initial indentation according to the Hertz model. The viscoelastic properties were extracted by fitting the stress-relaxation curve. (C) Representative stress-relaxation curves and theoretical fitting of chondrocytes from variable stiffness substrates during the cell swelling, respectively. A higher coefficient of determination (R2) indicates a better match between the stress relaxation model and experimental data. (D-G) Comparison of mechanical parameters of chondrocytes on varia-ble stiffness substrates during the cell swelling. Stiff substrate markedly increased the mechanical parameters (Eelastic, ER, EO and $\mu$ ) of chondrocytes. (H-K) Comparison of mechanical pa-rameters of chondrocytes on variable stiffness substrates in the iso-osmotic ( $320 \mathrm{mOsm}$ ) (control group) and hypoosmotic (180 mOsm) medium. N.S., not significant ( $p>0.05) .{ }^{*}, p<0.05 ; * \star, p<0.01 ; * \star \star, p<0.001$; one-way ANOVA and post hoc Tukey's test. In each panel, bar height denotes the mean of the data points, error bars denote standard error of the mean; the number of cells analyzed from variable stiffness substrates is specified above each bar. 
A

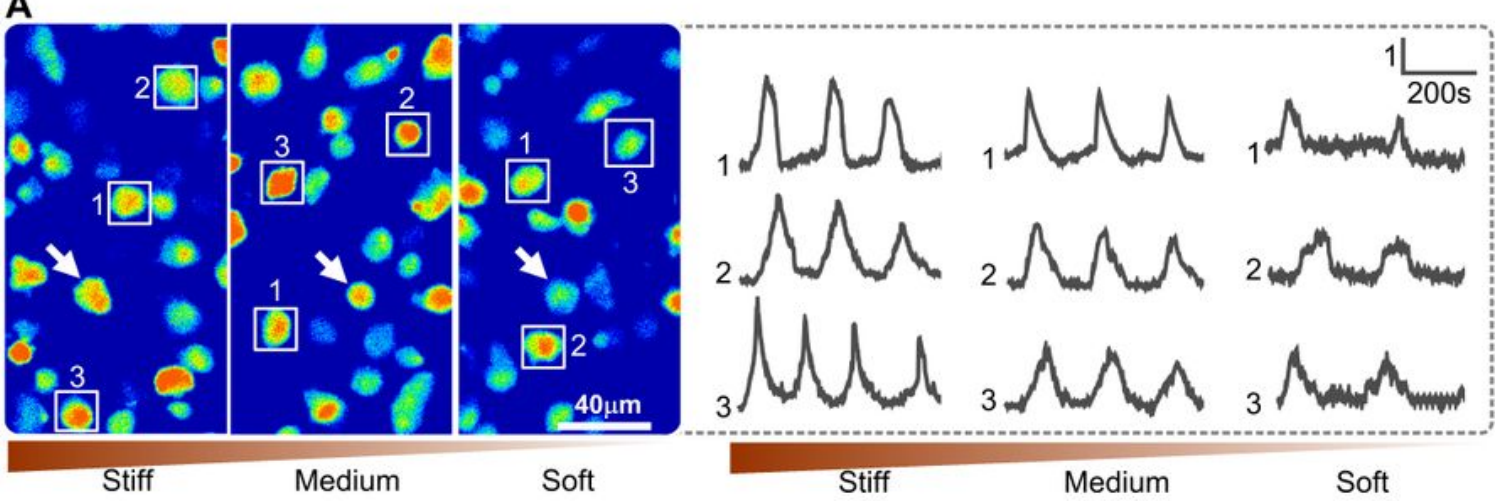

B

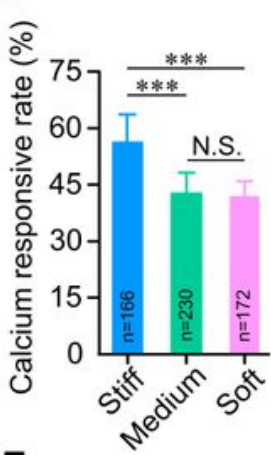

C

E
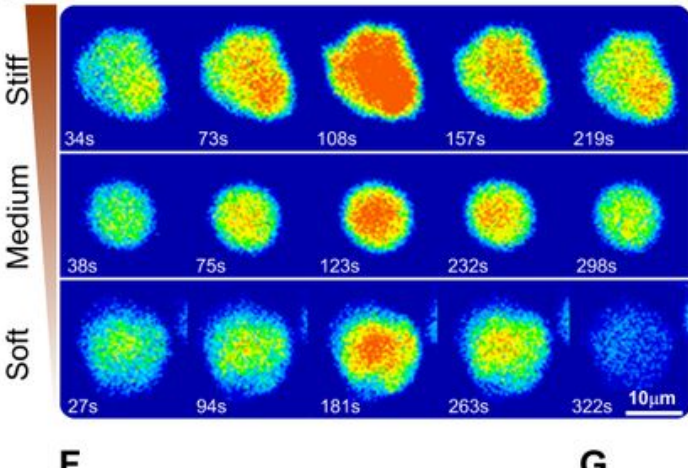

D

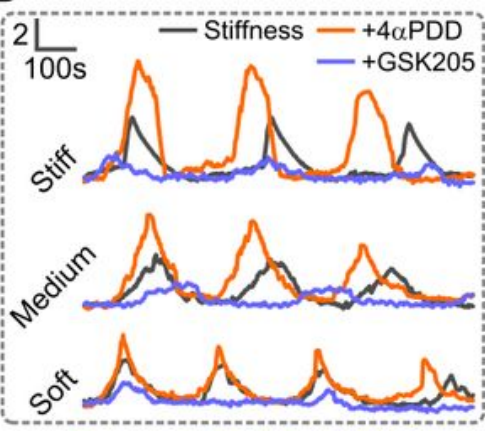

F

G

H
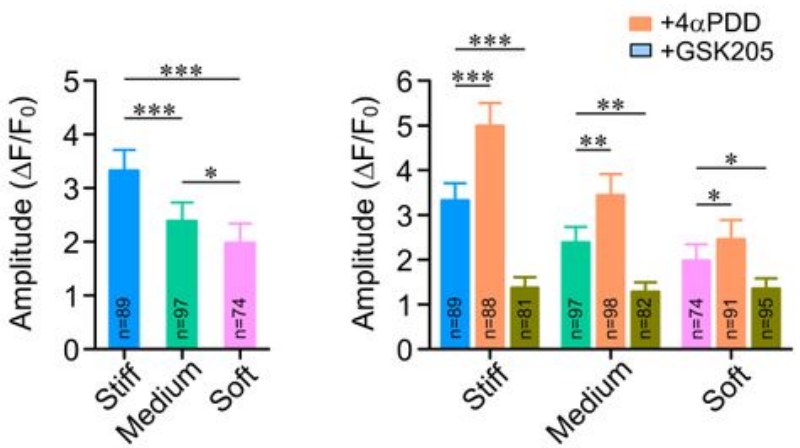

G +...+4APDD

.......SK205

I

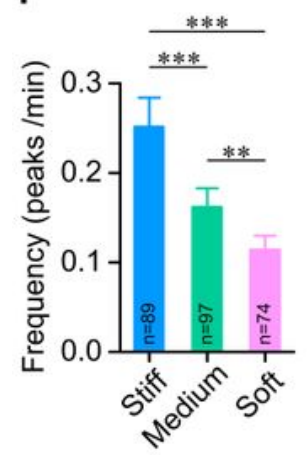

J
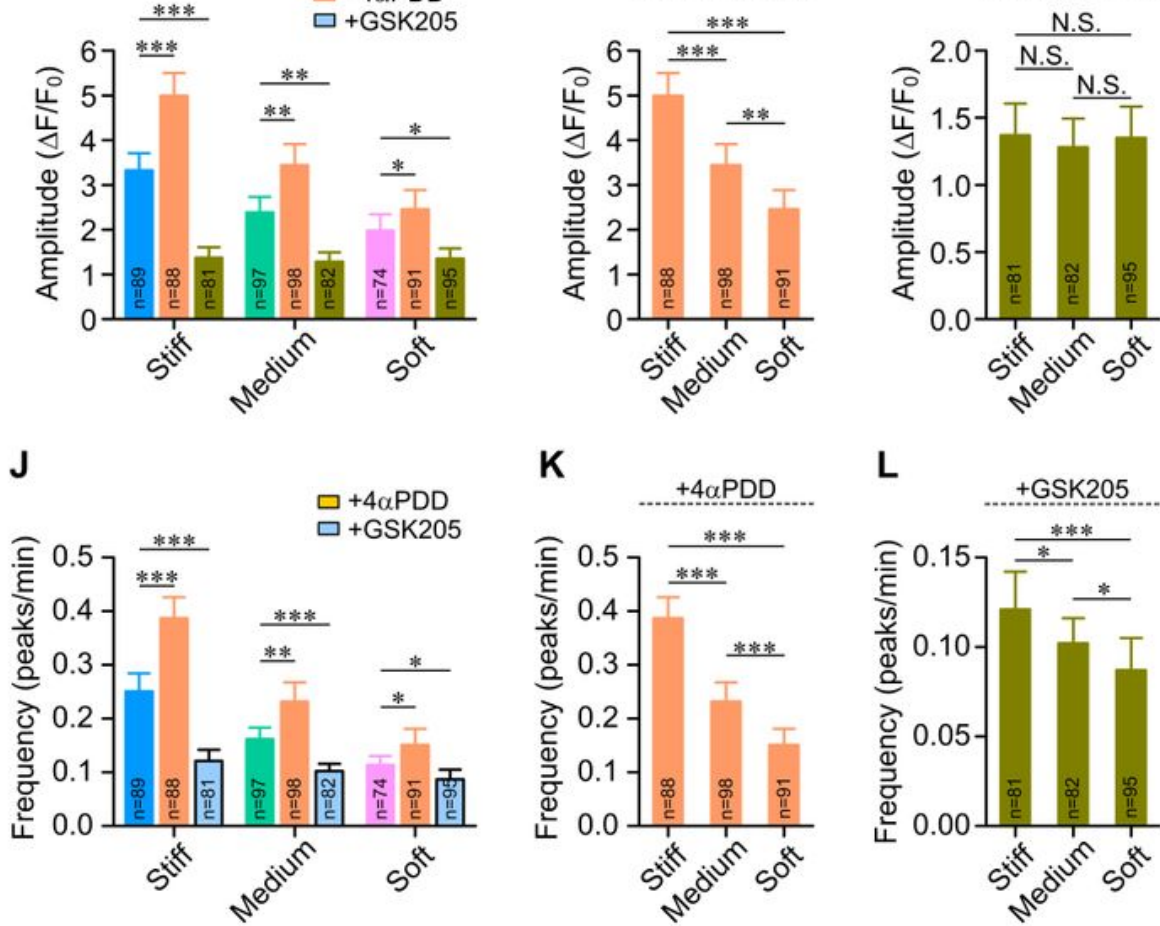

L

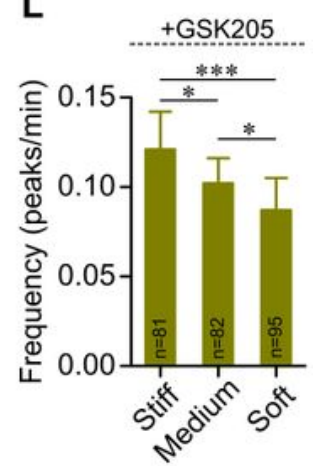

Figure 4

The effects of substrate stiffness on cytosolic Ca2+ oscillations in chondrocytes in iso-osmotic medium (320 mOsm). (A) Representative cell images showing Ca2+ fluorescence in chondro-cytes on variable stiffness substrates, as marked with square and white arrow in figures. Time elapse recordings of $\mathrm{Ca} 2+$ oscillations of three chondrocytes on the stiff, medium and soft sub-strates, respectively, as denoted by the square in corresponding figure 4A (right). (B) The per-centage of $\mathrm{Ca} 2+$ oscillation in chondrocyte was 
significantly increased on the stiff substrates. The calcium responsive rate of chondrocytes on stiff, medium and soft substrate in iso-osmotic me-dium (320 mOsm) was $56 \pm 8 \%, 43 \pm 6 \%$ and $42 \pm 4 \%$, respectively. (C) Representative three chondrocyte showing cytosolic $\mathrm{Ca} 2+$ oscillations from the stiff, medium and soft substrate, re-spectively, as marked with white arrows in figure 4A. (D) Representative time elapse recordings of $\mathrm{Ca} 2+$ oscillations of chondrocytes on the stiff, medium and soft substrates treated by TRPV4 agonist 4aPDD and TRPV4 antagonist GSK205. (E) The amplitude of Ca2+ oscillations in chon-drocytes was significantly higher on stiff substrate than soft substrates. (F) Comparison of the amplitude of $\mathrm{Ca} 2+$ oscillations in chondrocytes on variable stiffness substrates with or without pharmacologically treatment. (G) The amplitude of $\mathrm{Ca} 2+$ oscillations in chondrocytes activated by TRPV4 agonist 4aPDD was significantly higher on the stiff substrate than soft substrate. $(H)$ The amplitude of $\mathrm{Ca} 2+$ oscillations in chondrocytes on varying substrate stiffness inhibited by TRPV4 antagonist GSK205 has no significant difference. (I) The frequency of Ca2+ oscillations in chondrocytes was significantly higher on the stiff substrate than soft substrate. $(\mathrm{J})$ Comparison of the frequency of $\mathrm{Ca} 2+$ oscillations in chondrocytes on substrates of varying stiffness with or without pharmacologically treatment. (K) The frequency of Ca2+ oscillations in chondrocytes activated by 4aPDD was significantly higher on the stiff substrate than the soft substrate. $(L)$ The frequency of Ca2+ oscillations in chondrocytes inhibited by GSK205 was significantly higher on the stiff substrate than soft substrate. The cytosolic $\mathrm{Ca} 2+$ oscillations in chondrocytes were medi-ated by TRPV4 in substrate stiffness-dependent manner. N.S., not significant $(p>0.05) .{ }^{*}, p<0.05 ; * \star, p<0.01 ; \star \star \star, p<0.001$; one-way ANOVA and post hoc Tukey's test. 
A

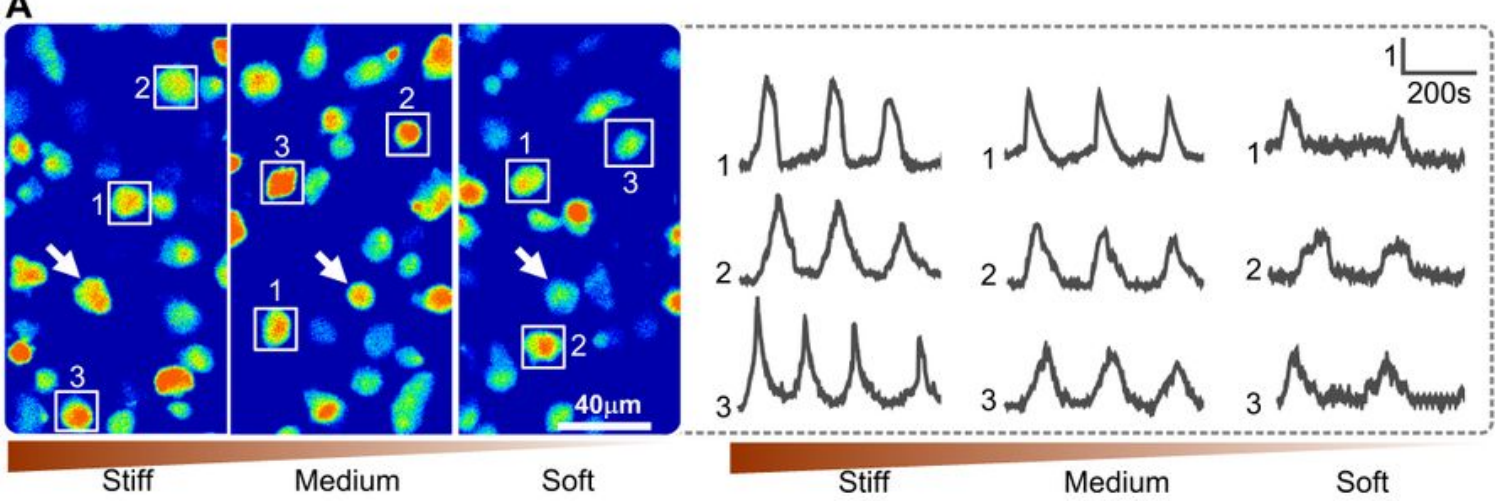

B

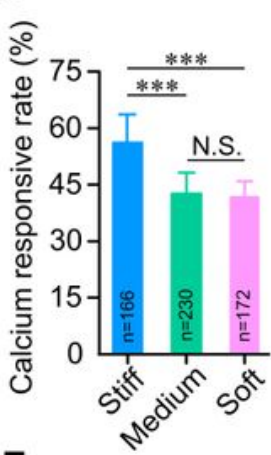

C

E
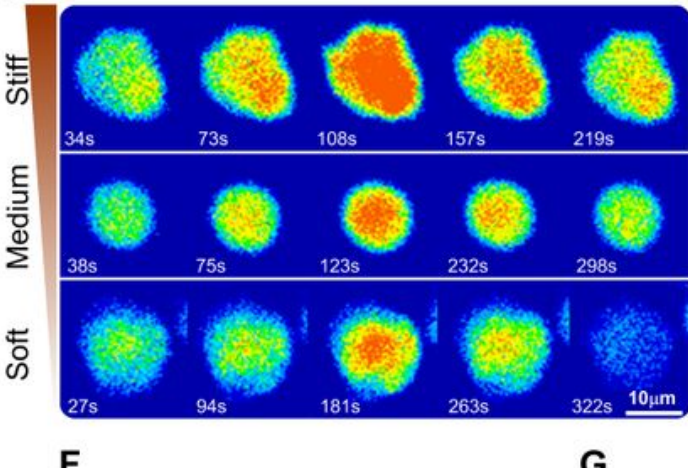

D

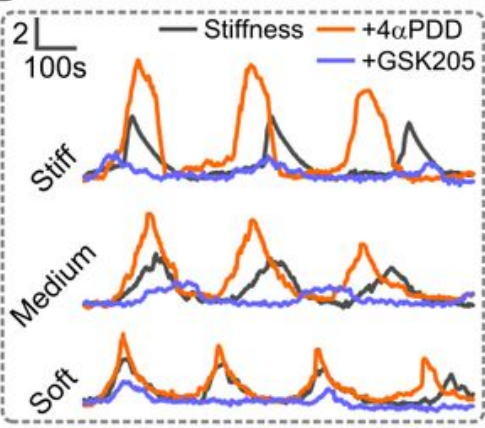

F

G

H
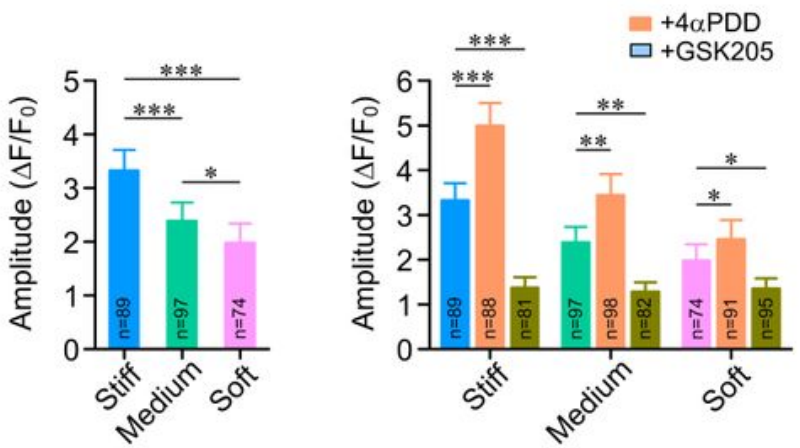

G +...+4APDD

.....+GSK205

I

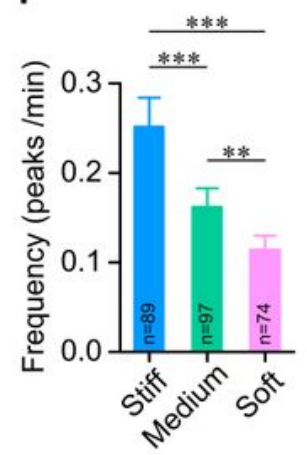

J
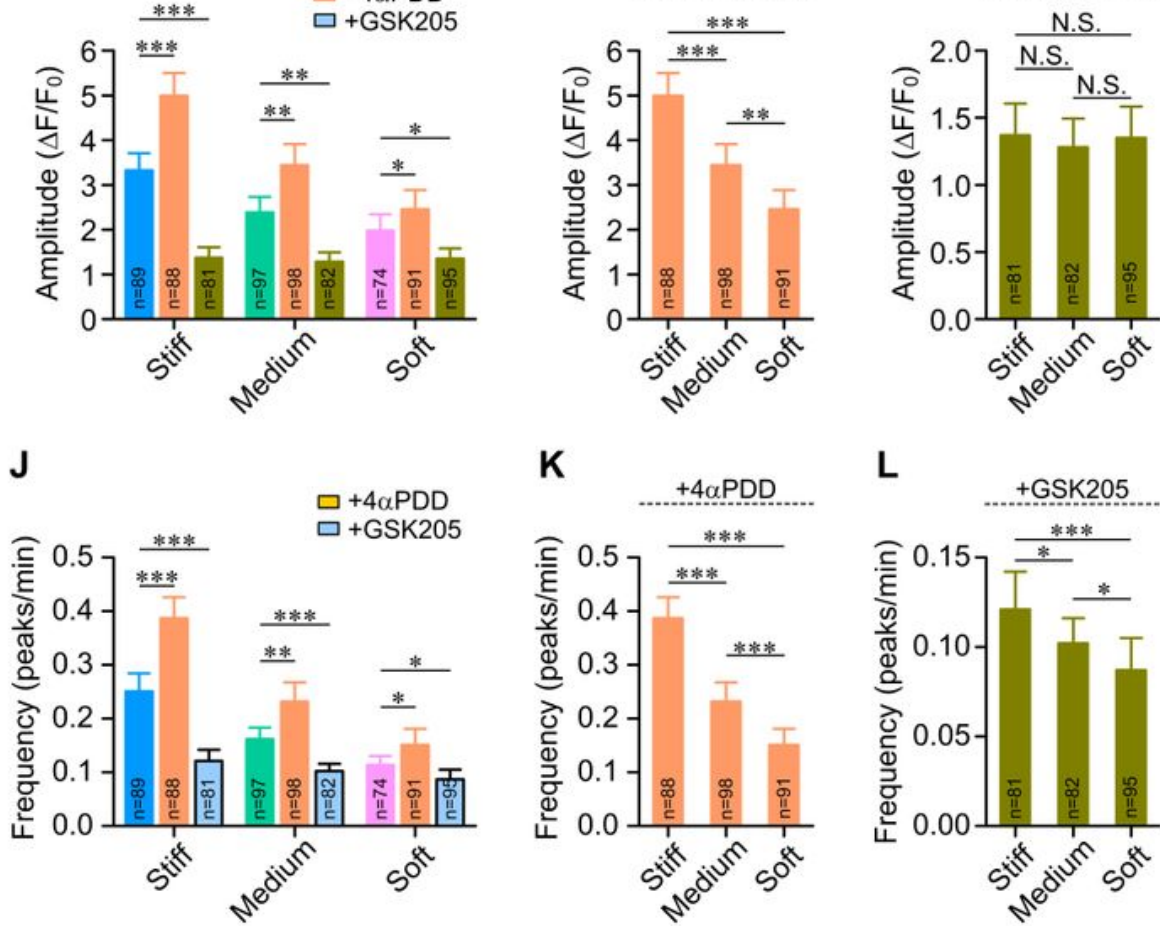

L

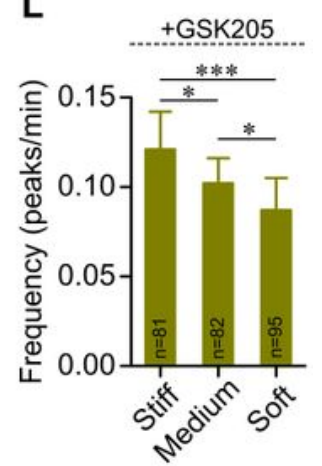

Figure 4

The effects of substrate stiffness on cytosolic Ca2+ oscillations in chondrocytes in iso-osmotic medium (320 mOsm). (A) Representative cell images showing Ca2+ fluorescence in chondro-cytes on variable stiffness substrates, as marked with square and white arrow in figures. Time elapse recordings of $\mathrm{Ca} 2+$ oscillations of three chondrocytes on the stiff, medium and soft sub-strates, respectively, as denoted by the square in corresponding figure 4A (right). (B) The per-centage of $\mathrm{Ca} 2+$ oscillation in chondrocyte was 
significantly increased on the stiff substrates. The calcium responsive rate of chondrocytes on stiff, medium and soft substrate in iso-osmotic me-dium (320 mOsm) was $56 \pm 8 \%, 43 \pm 6 \%$ and $42 \pm 4 \%$, respectively. (C) Representative three chondrocyte showing cytosolic $\mathrm{Ca} 2+$ oscillations from the stiff, medium and soft substrate, re-spectively, as marked with white arrows in figure 4A. (D) Representative time elapse recordings of $\mathrm{Ca} 2+$ oscillations of chondrocytes on the stiff, medium and soft substrates treated by TRPV4 agonist 4aPDD and TRPV4 antagonist GSK205. (E) The amplitude of Ca2+ oscillations in chon-drocytes was significantly higher on stiff substrate than soft substrates. (F) Comparison of the amplitude of $\mathrm{Ca} 2+$ oscillations in chondrocytes on variable stiffness substrates with or without pharmacologically treatment. (G) The amplitude of $\mathrm{Ca} 2+$ oscillations in chondrocytes activated by TRPV4 agonist 4aPDD was significantly higher on the stiff substrate than soft substrate. $(H)$ The amplitude of $\mathrm{Ca} 2+$ oscillations in chondrocytes on varying substrate stiffness inhibited by TRPV4 antagonist GSK205 has no significant difference. (I) The frequency of Ca2+ oscillations in chondrocytes was significantly higher on the stiff substrate than soft substrate. $(\mathrm{J})$ Comparison of the frequency of $\mathrm{Ca} 2+$ oscillations in chondrocytes on substrates of varying stiffness with or without pharmacologically treatment. (K) The frequency of Ca2+ oscillations in chondrocytes activated by 4aPDD was significantly higher on the stiff substrate than the soft substrate. $(L)$ The frequency of Ca2+ oscillations in chondrocytes inhibited by GSK205 was significantly higher on the stiff substrate than soft substrate. The cytosolic $\mathrm{Ca} 2+$ oscillations in chondrocytes were medi-ated by TRPV4 in substrate stiffness-dependent manner. N.S., not significant $(p>0.05) .{ }^{*}, p<0.05 ; * \star, p<0.01 ; \star \star \star, p<0.001$; one-way ANOVA and post hoc Tukey's test. 
A

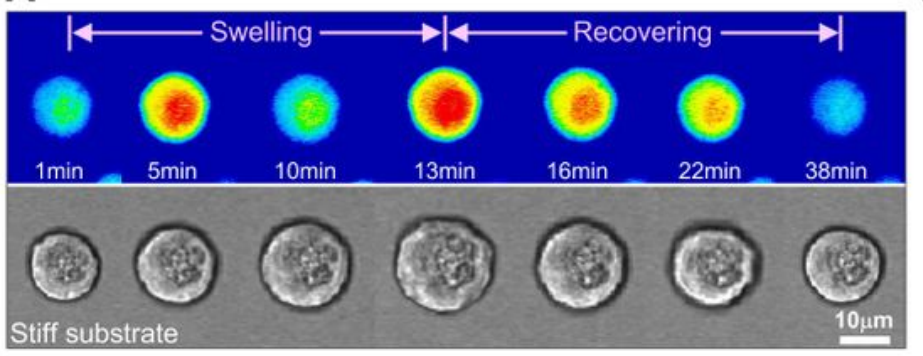

C

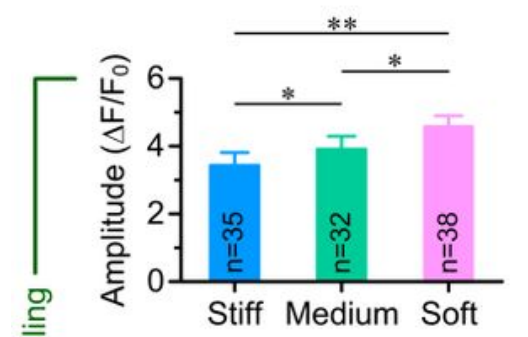

F

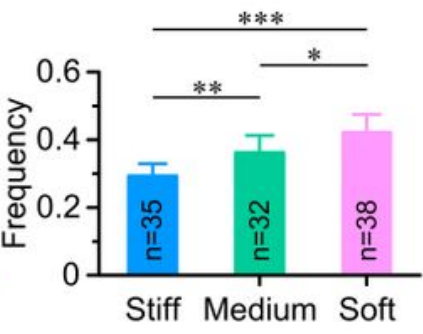

I

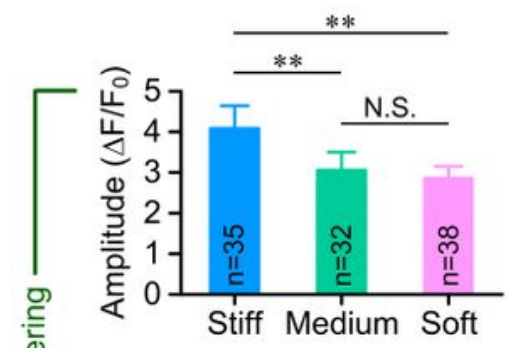

L

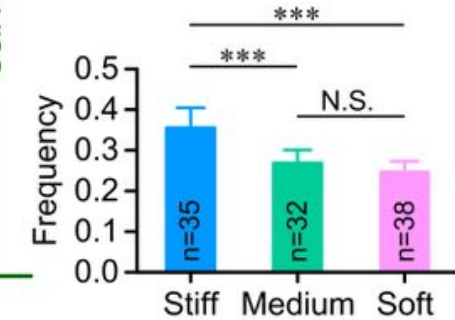

D

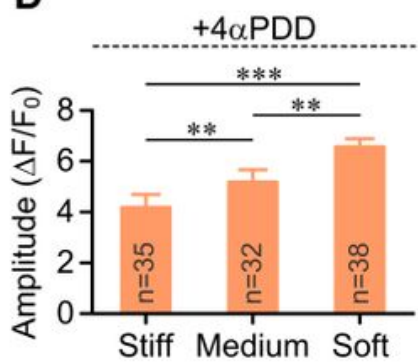

G
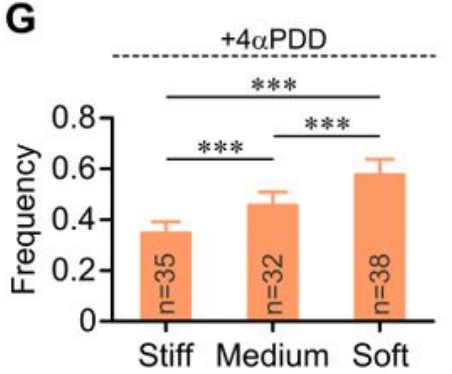

J
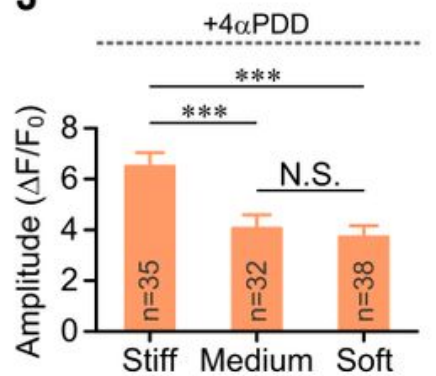

M

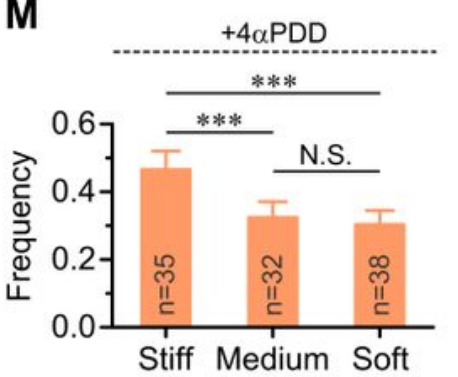

B $200 \mathrm{~s}$
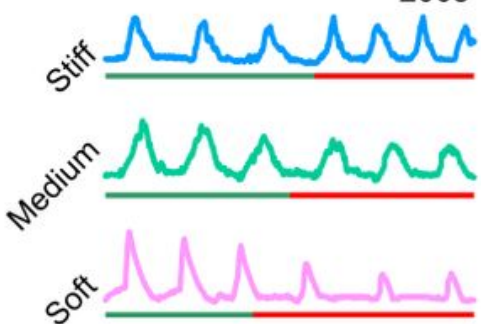

$E$

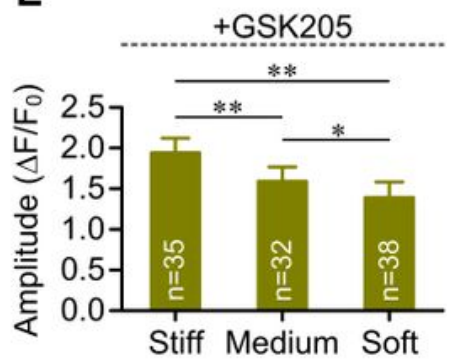

H

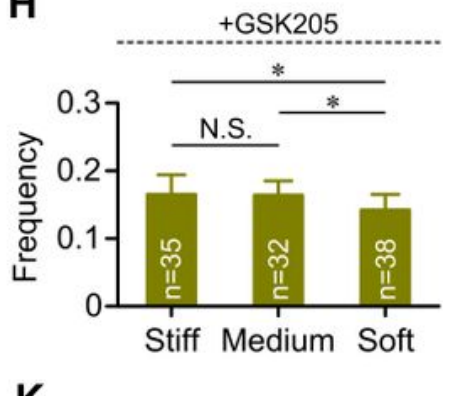

K

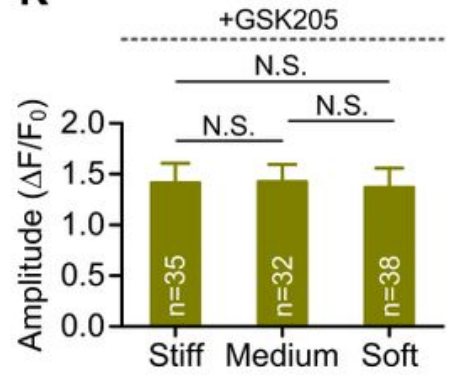

$\mathbf{N}$

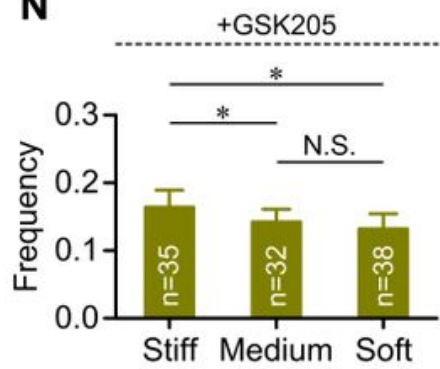

\section{Figure 5}

Quantitative analysis of Ca2+ oscillations in chondrocytes on variable stiffness substrates in hy-poosmotic medium (180 mOsm). (A) Representative single-cell images showing Ca2+ fluores-cence in chondrocyte on the stiff substrate during the RVD response. (B) Representative time-elapse recordings of $\mathrm{Ca} 2+$ oscillations in chondrocyte on the stiff, medium and soft substrates in the cell RVD response, respectively. The green lines represent the cell swelling process and the red lines represent the cell 
recovering process. (C-H) Summary of the amplitude and frequency of cytosolic Ca2+ oscillations in chondrocytes on varying substrate stiffness with or without TRPV4 agonist 4aPDD or TRPV4 antagonist GSK205 during cell swelling. The amplitude and frequency of cytosolic $\mathrm{Ca} 2+$ oscillations in chondrocytes were significantly higher on the soft substrate than stiff substrate during the cell swelling. (I-N) Summary of the amplitude and fre-quency of cytosolic $\mathrm{Ca} 2+$ oscillations in chondrocytes on varying substrate stiffness with or without TRPV4 agonist 4aPDD or TRPV4 antagonist GSK205 during the cell recovering. The amplitude and frequency of cytosolic $\mathrm{Ca} 2+$ oscillations in chondrocytes were significantly higher on the stiff substrate than softer substrate. The spontaneous cytosolic Ca2+ oscillations in chon-drocytes were mediated by TRPV4 in substrate stiffness-dependent manner during cell RVD re-sponse. The numbers shown in parenthesis indicate the number of cells analyzed per substrate stiffness. N.S., not significant $(p>0.05) .{ }^{*}, p<0.05 ; * \star, p<0.01 ; * \star \star, p<0.001$; one-way ANOVA and post hoc Tukey's test. 
A

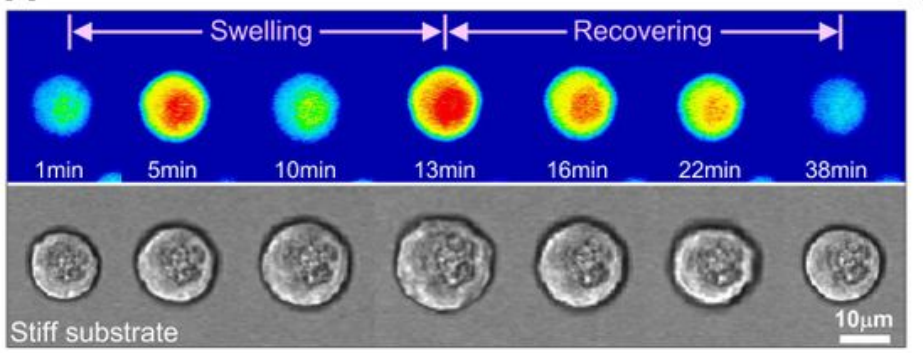

C

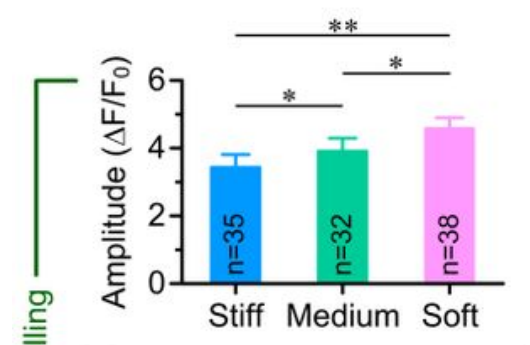

F

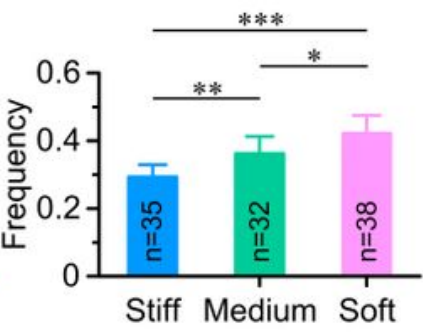

I

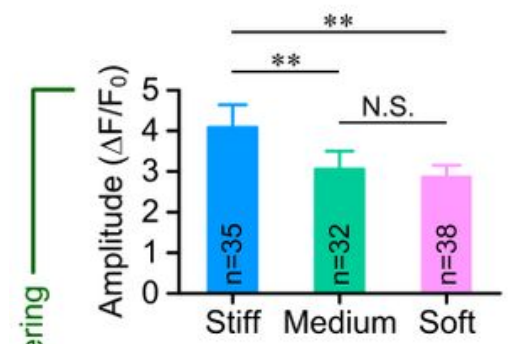

L

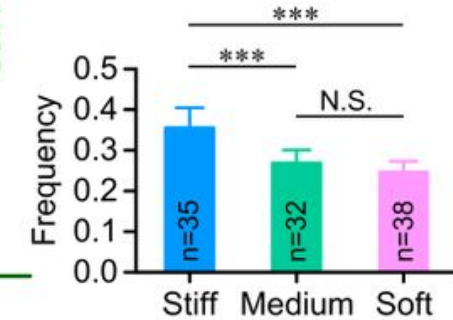

D

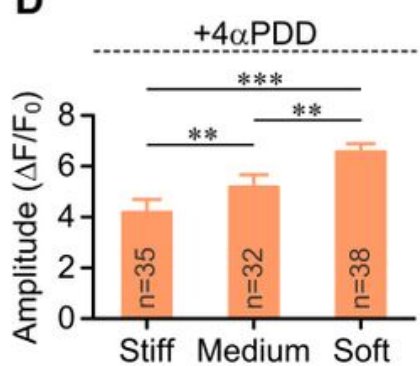

G
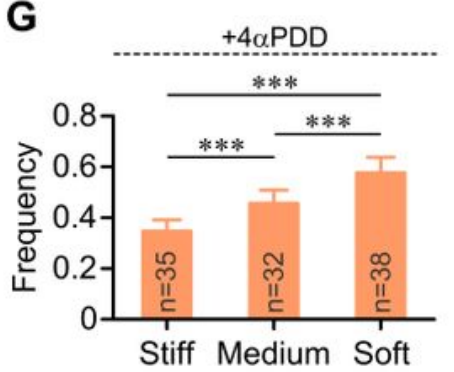

J
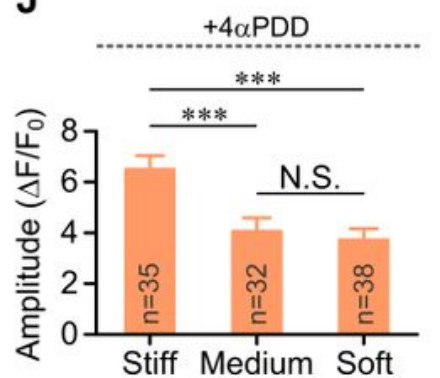

M

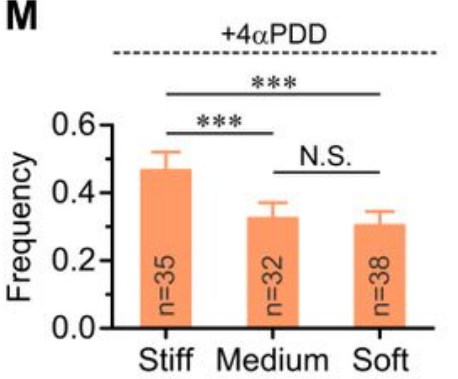

B

2 200s

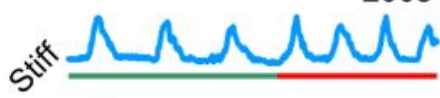

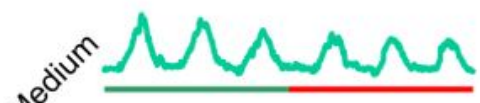

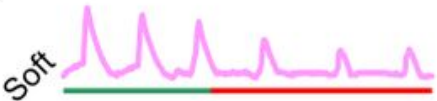

E

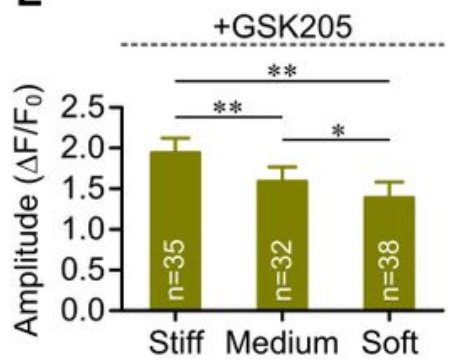

H

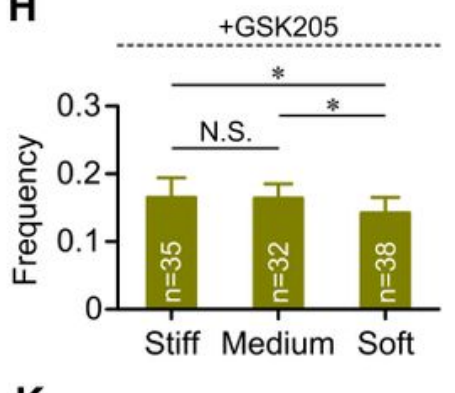

K

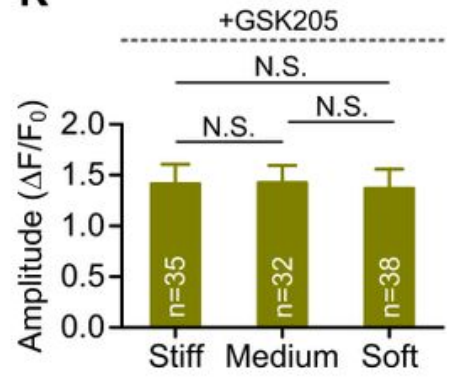

$\mathbf{N}$

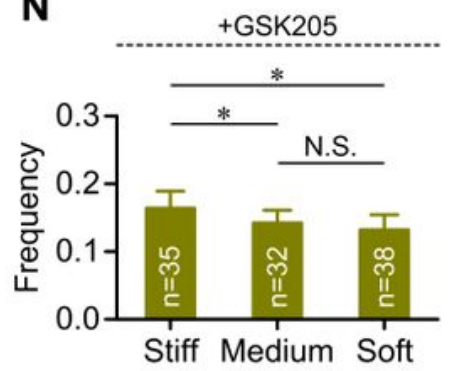

\section{Figure 5}

Quantitative analysis of Ca2+ oscillations in chondrocytes on variable stiffness substrates in hy-poosmotic medium (180 mOsm). (A) Representative single-cell images showing $\mathrm{Ca} 2+$ fluores-cence in chondrocyte on the stiff substrate during the RVD response. (B) Representative time-elapse recordings of $\mathrm{Ca} 2+$ oscillations in chondrocyte on the stiff, medium and soft substrates in the cell RVD response, respectively. The green lines represent the cell swelling process and the red lines represent the cell 
recovering process. (C-H) Summary of the amplitude and frequency of cytosolic Ca2+ oscillations in chondrocytes on varying substrate stiffness with or without TRPV4 agonist 4aPDD or TRPV4 antagonist GSK205 during cell swelling. The amplitude and frequency of cytosolic $\mathrm{Ca} 2+$ oscillations in chondrocytes were significantly higher on the soft substrate than stiff substrate during the cell swelling. (I-N) Summary of the amplitude and fre-quency of cytosolic $\mathrm{Ca} 2+$ oscillations in chondrocytes on varying substrate stiffness with or without TRPV4 agonist 4aPDD or TRPV4 antagonist GSK205 during the cell recovering. The amplitude and frequency of cytosolic $\mathrm{Ca} 2+$ oscillations in chondrocytes were significantly higher on the stiff substrate than softer substrate. The spontaneous cytosolic Ca2+ oscillations in chon-drocytes were mediated by TRPV4 in substrate stiffness-dependent manner during cell RVD re-sponse. The numbers shown in parenthesis indicate the number of cells analyzed per substrate stiffness. N.S., not significant $(p>0.05) .{ }^{*}, p<0.05 ; * \star, p<0.01 ; * \star \star, p<0.001$; one-way ANOVA and post hoc Tukey's test. 

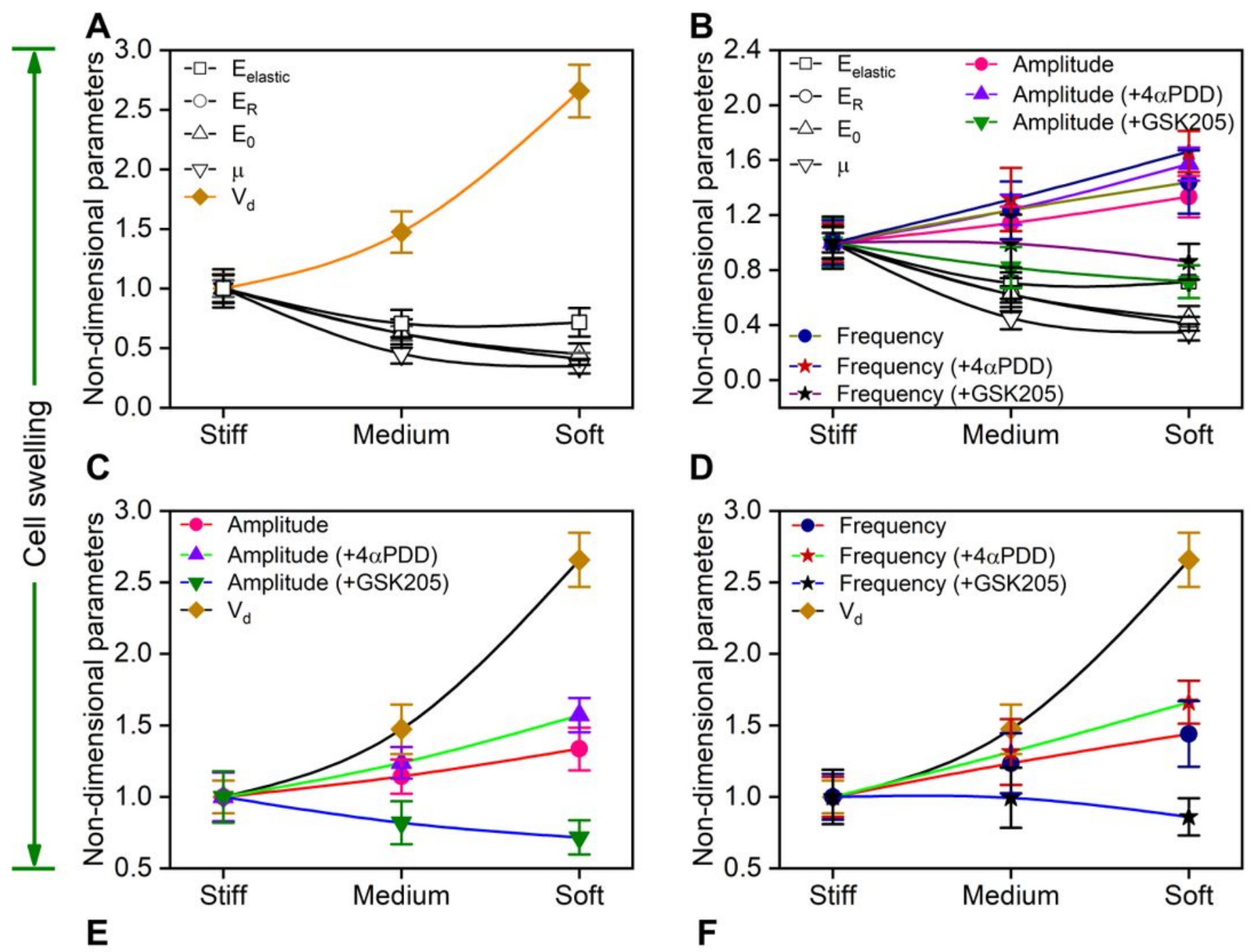

D
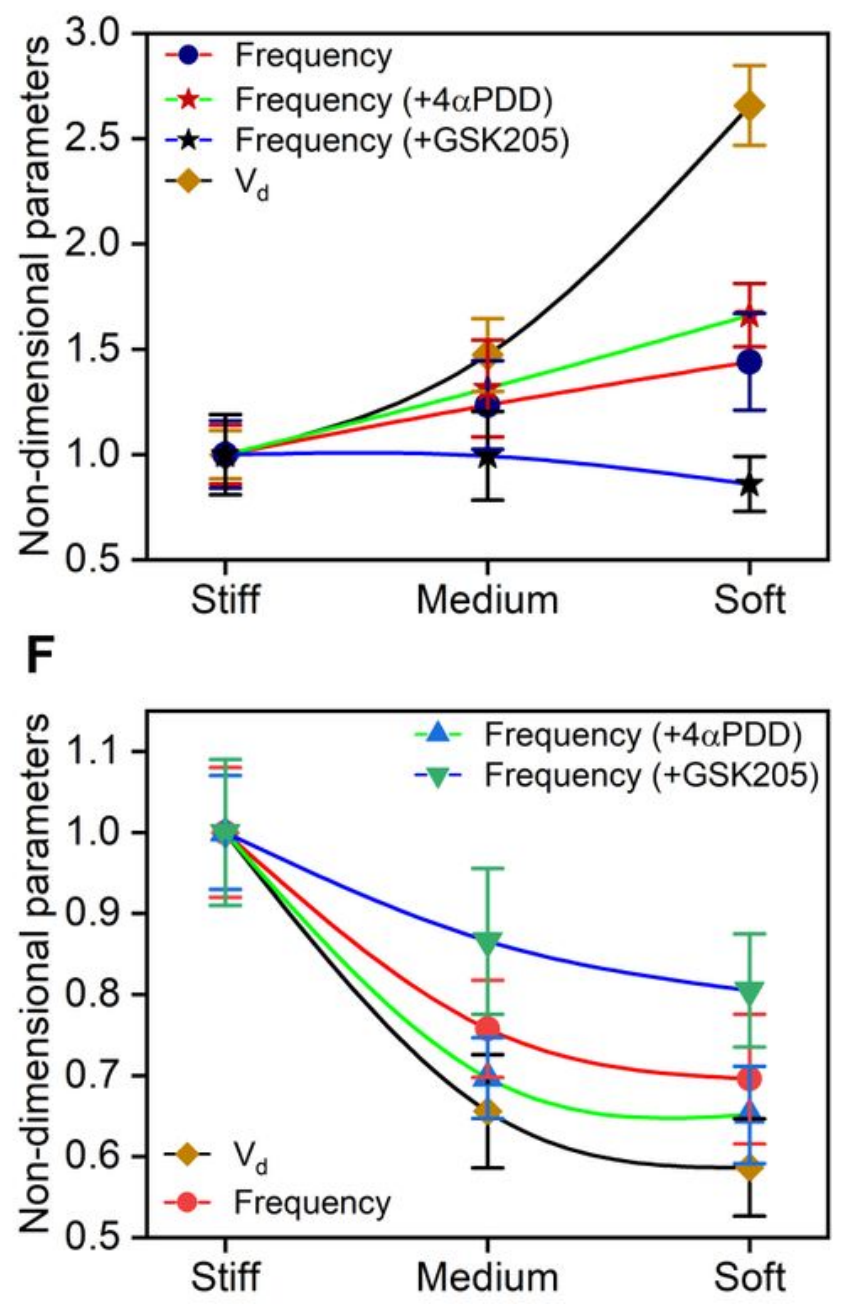

\section{Figure 6}

The relationship between non-dimensional mechanical parameters, RVD response parameters and cytosolic $\mathrm{Ca} 2+$ oscillations parameters in chondrocytes on variable stiffness substrates during the cell swelling and recovering. 

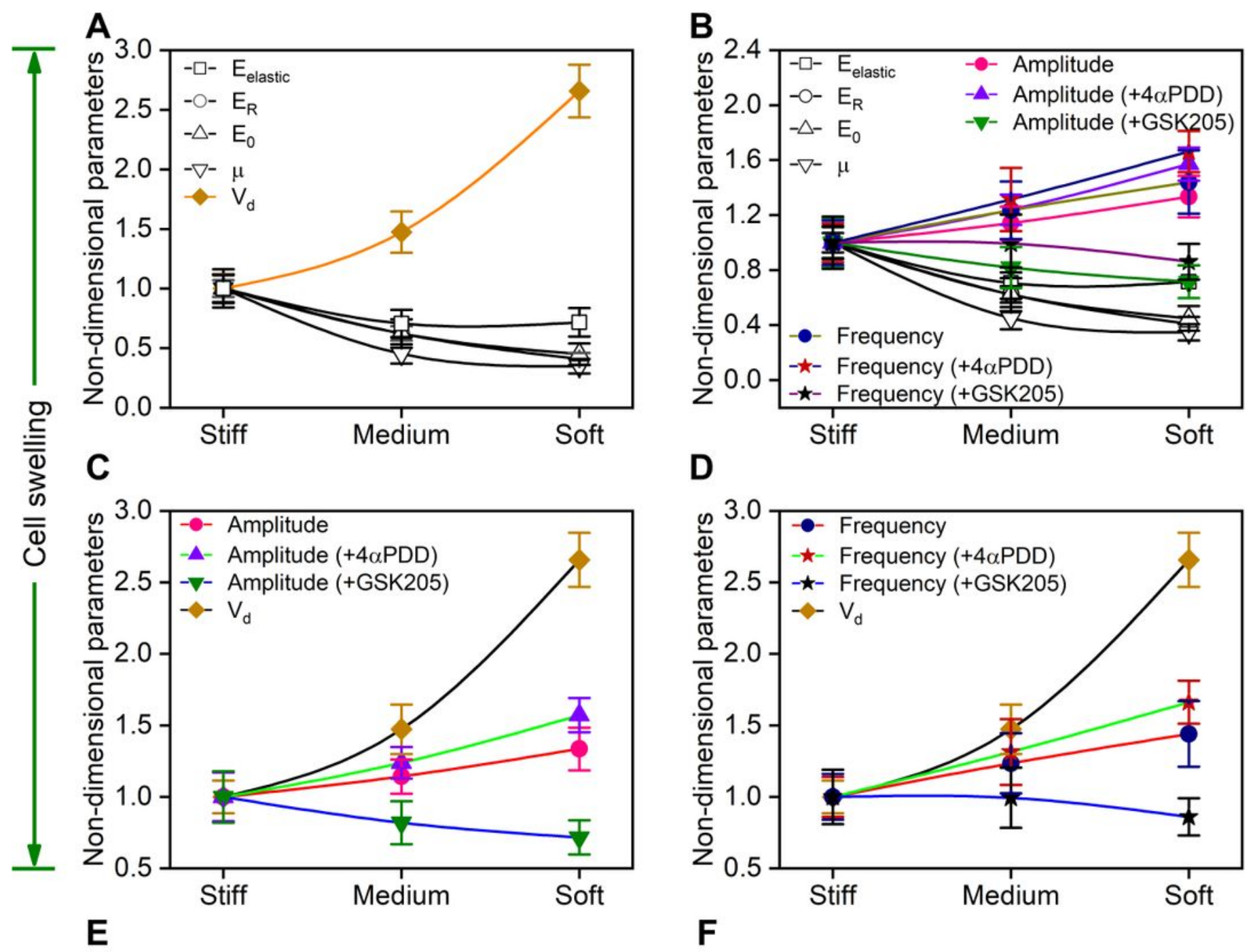

D

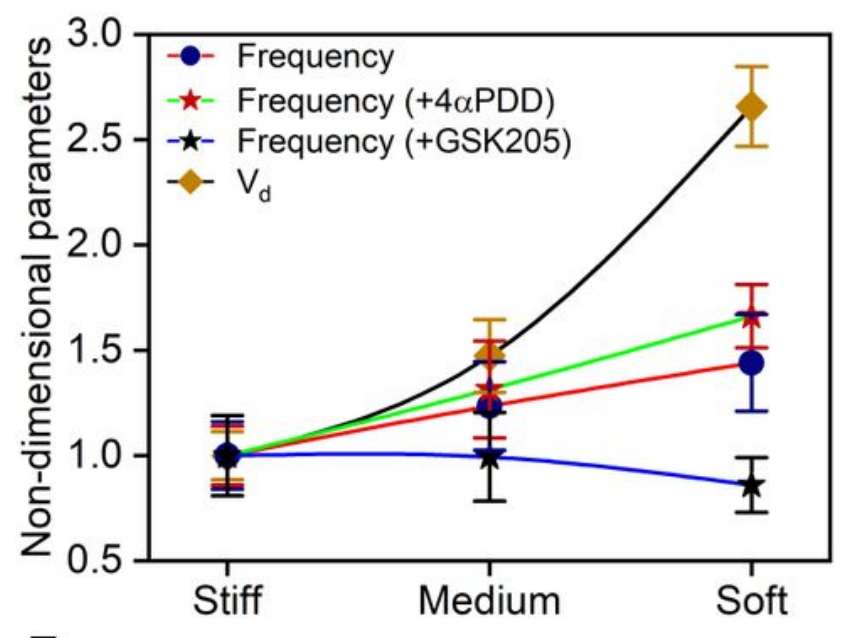

$\mathbf{F}$
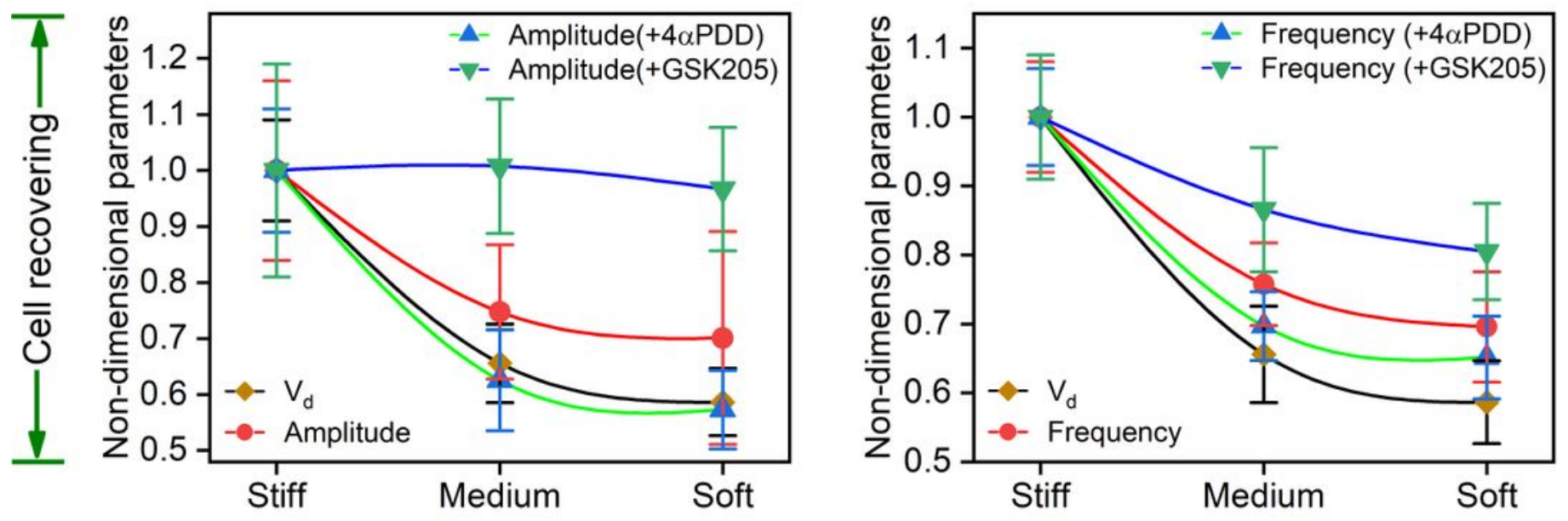

Figure 6

The relationship between non-dimensional mechanical parameters, RVD response parameters and cytosolic $\mathrm{Ca} 2+$ oscillations parameters in chondrocytes on variable stiffness substrates during the cell swelling and recovering. 
This is a list of supplementary files associated with this preprint. Click to download.

- Table1.JPG

- Table1.JPG

- SRSupplementMaterial29Nov2020byZhangQY.docx

- SRSupplementMaterial29Nov2020byZhangQY.docx 